\title{
Solving Parameterized Linear Difference Equations In Terms of Indefinite Nested Sums and Products
}

\author{
Carsten Schneider \\ Research Institute for Symbolic Computation \\ J. Kepler University Linz \\ A-4040 Linz, Austria \\ Carsten.Schneider@risc.uni-linz.ac.at \\ (11 November 2004)
}

\begin{abstract}
The described algorithms enable one to find all solutions of parameterized linear difference equations within $\Pi \Sigma$-fields, a very general class of difference fields. These algorithms can be applied to a very general class of multisums, for instance, for proving identities and simplifying expressions.
\end{abstract}

Keywords: symbolic summation, difference fields, $\Pi \Sigma$-extensions, $\Pi \Sigma$-fields

AMS Subject Classification: 33FXX, 68W30, 12H10

\section{Introduction}

Solving parameterized linear difference equations (problem $P L D E$ ) covers various prominent subproblems in symbolic summation [1]. For instance, by using $P L D E$-solvers for the rational case [2-6] or its $q$-analog version [7] one can find sum solutions of $(q-)$ difference equations, see [8-10], or one can deal with telescoping and creative telescoping for $\partial$-finite summand expressions, see [11]. Moreover, telescoping and creative telescoping algorithms for $\left(q^{-}\right)$hypergeometric terms, like [12-16], or its mixed case, like [17], are nothing else than special purpose solvers for certain instances of problem PLDE.

More generally, in [18] algorithms have been developed that solve the first order case of problem PLDE for $\Pi \Sigma$-extensions [19]. Within these difference fields one cannot only consider $(q-)$ hypergeometric terms, see [20], but rational terms consisting of arbitrarily nested indefinite sums and products; see [21]. Karr's algorithm is, in a sense, the summation counterpart of Risch's algorithm [22] for indefinite integration.

Supported by the SFB-grant F1305 and the grant P16613-N12 of the Austrian FWF. 
Another approach is [23] where one can try to solve problem PLDE for a subclass of monomial extensions that covers besides indefinite nested products (П-extensions) also differential fields; see also [24]. The only restriction is that one cannot consider indefinite nested sums and products ( $\Pi \Sigma$-extensions) that arise frequently in symbolic summation.

In this article we shall develop a general framework that can treat problem $P L D E$ for this important class of $\Pi \Sigma$-extensions. More precisely, we shall derive the following results.

- We obtain a simplified and streamlined version of Karr's algorithm, see Theorem 4.7 , by using results from $[23,25,26]$. Based on this we were able to develop extended summation algorithms in [27-29].

- We generalize the reduction techniques presented in [18] from the first order to the higher order case. This gives an algorithm, see Theorem 4.2, that solves problem $P L D E$ for unimonomial and $\Pi \Sigma$-extensions if certain subproblems can be solved in the ground field.

- For general $\Pi \Sigma$-extensions and $\Pi \Sigma$-fields there are still some building blocks missing to turn our method to a complete algorithm. More precisely, there are no algorithms so far which determine a common denominator of all the rational solutions and which bound the degree of the numerator of those solutions. However, there are algorithms that can approximate those bounds in $\Pi \Sigma$-fields. This allows us to search systematically for all solutions by increasing step by step the domain of the possible solutions. We show that after finitely many steps one eventually finds all solutions; see Theorem 5.7.

Our new methods significantly enhance the summation approaches mentioned above or given in [30,31]. Namely, we can handle telescoping, creative telescoping and recurrence solving in $\Pi \Sigma$-extensions; see [32]. Moreover, we can apply telescoping and creative telescoping for $\partial$-finite expressions in terms of $\Pi \Sigma$-extensions; see [33].

All these methods are implemented in the summation package Sigma, which is based on the computer algebra system Mathematica. The wide applicability of Sigma is illustrated for instance in [10,33-37]. We will illustrate our results by non-trivial examples from [33] and [37] throughout this paper.

The general structure is as follows. In Section 2 we supplement the key problem $P L D E$ by various illustrative examples. In Section 3 we present reduction strategies for problem $P L D E$ in unimonomial and $\Pi \Sigma$-extensions. In Section 4 we present the corresponding algorithm which depends on the two subproblems DenB and DegB; these problems have not been solved for general $\Pi \Sigma$-fields so far. In Section 5 we introduce a weakened version that does not rely on the problems $\operatorname{Den} B$ and $\operatorname{Deg} B$, but only on problem $W D e n B$; this problem can be solved for general $\Pi \Sigma$-fields. The resulting algorithm enables us to search systematically for all solutions of problem $P L D E$ in $\Pi \Sigma$-fields. 


\section{Parameterized linear difference equations and symbolic summation}

Let $(\mathbb{F}, \sigma)$ be a difference field, i.e., a field ${ }^{1} \mathbb{F}$ together with a field automorphism $\sigma: \mathbb{F} \rightarrow \mathbb{F}$. Furthermore, define the constant field $\mathbb{K}$ of $(\mathbb{F}, \sigma)$ by $\mathbb{K}=$ const $_{\sigma} \mathbb{F}:=\{k \in \mathbb{F} \mid \sigma(k)=k\}$. Then we are interested in the following problem $^{2}$.

\section{PLDE: Parameterized Linear Difference Equations.}

- Given $(\mathbb{F}, \sigma)$ with $\mathbb{K}:=$ const $_{\sigma} \mathbb{F}, \mathbf{0} \neq \boldsymbol{a}=\left(a_{1} \ldots, a_{m}\right) \in \mathbb{F}^{m}, \boldsymbol{f}=\left(f_{1}, \ldots, f_{n}\right) \in \mathbb{F}^{n}$.

- Find all $g \in \mathbb{F}$ and $\left(c_{1}, \ldots, c_{n}\right) \in \mathbb{K}^{n}$ with

$$
a_{1} \sigma^{m-1}(g)+\cdots+a_{m} g=c_{1} f_{1}+\cdots+c_{n} f_{n} .
$$

Note that in any difference field $(\mathbb{F}, \sigma)$ with $\mathbb{K}:=$ const $_{\sigma} \mathbb{F}$, the field $\mathbb{F}$ can be interpreted as a vector space over $\mathbb{K}$. Hence problem $P L D E$ can be described by the following set.

Definition 2.1 Let $(\mathbb{F}, \sigma)$ be a difference field with $\mathbb{K}:=$ const $_{\sigma} \mathbb{F}$ and $\mathbb{V}$ be a subspace of $\mathbb{F}$ over $\mathbb{K}$. Let $\mathbf{0} \neq \boldsymbol{a}=\left(a_{1}, \ldots, a_{m}\right) \in \mathbb{F}^{m}$ and $\boldsymbol{f}=\left(f_{1}, \ldots, f_{n}\right) \in$ $\mathbb{F}^{n}$. We define the solution space for $\boldsymbol{a}, \boldsymbol{f}$ in $\mathbb{V}$ by $\mathrm{V}(\boldsymbol{a}, \boldsymbol{f}, \mathbb{V})=\left\{\left(c_{1}, \ldots, c_{n}, g\right) \in\right.$ $\mathbb{K}^{n} \times \mathbb{V}:$ (1) holds .

It is easy to see that $\mathrm{V}(\boldsymbol{a}, \boldsymbol{f}, \mathbb{V})$ is a vector space over $\mathbb{K}$. Moreover, in [39] based on [40, Thm. XII (page 272)] it is proven that the dimension of this vector space is at most $m+n-1$. Summarizing, problem $P L D E$ is equivalent to finding a basis of $\mathrm{V}(\boldsymbol{a}, \boldsymbol{f}, \mathbb{F})$.

So far, various $P L D E$-solvers have been developed for symbolic summation, like the algorithms in $[2-6]$ for the rational case, i.e., $\mathbb{F}=\mathbb{K}(k)$ with $\sigma(k)=k+1$, or the algorithms in [7] for the $q$-analogue version, i.e., $\mathbb{F}=\mathbb{K}(q)(x)$ with $q$ transcendental over $\mathbb{K}$ and $\sigma(x)=q x$. Besides this, special purpose solvers have been developed for telescoping and creative telescoping for $\left(q^{-}\right)$hypergeometric terms, see $[12,13,15,16]$, and for mixed hypergeometric terms, see [17]. Moreover, by using the methods in [23] one can attack problem $P L D E$ for $\Pi$-extensions.

In this article we complement all these approaches by considering problem $P L D E$ in $\Pi \Sigma$-extensions and $\Pi \Sigma$-fields. As illustrated in $[32,33]$ these algorithms substantially enhance the algorithmic tool box of symbolic summation.

Example 2.2 Consider the following elementary problem: Eliminate the sumquantifier in $\sum_{k=0}^{n} H_{k}$ where $H_{k}=\sum_{i=1}^{k} \frac{1}{i}$ denotes the $k$ th harmonic number.

\footnotetext{
${ }^{1}$ Throughout this paper all fields will have characteristic 0 .

${ }^{2}$ For the theory of difference equations in difference rings we refer to [38].
} 
In order to accomplish this task, we construct the difference field $(\mathbb{E}, \sigma)$ where $\mathbb{E}=\mathbb{Q}(k)(h)$ is a rational function field, and the field automorphism $\sigma: \mathbb{E} \rightarrow \mathbb{E}$ is uniquely defined by $\sigma(k)=k+1$ and $\sigma(h)=h+\frac{1}{k+1}$. Note that the shift $S_{k} H_{k}=H_{k}+\frac{1}{k+1}$ is reflected by the action of $\sigma$ on $h$. Given $(\mathbb{E}, \sigma)$, we compute by our algorithms, see Example 3.3, the solution $g=k t-k$ for $\sigma(g)-g=h$. Reinterpreting $g$ as the sequence $g(k)=k H_{k}-k$ we get the telescoping equation $g(k+1)-g(k)=H_{k}$. Summing this equation over $k$ from 0 to $n$ gives $\sum_{k=0}^{n} H_{k}=\left(H_{n+1}-1\right)(n+1)$.

Example 2.3 In [37] we have proved a family of identities including

$$
\sum_{k=0}^{n}\left(1-3(n-2 k) H_{k}\right)\left(\begin{array}{l}
n \\
k
\end{array}\right)^{3}=(-1)^{n}
$$

note that this family occurs in a generalized form in [41]. In order to find (2), we computed for the definite sum $S(n):=\sum_{k=0}^{n} f(n, k)$ with $f(n, k):=(1-$ $\left.3(n-2 k) H_{k}\right)\left(\begin{array}{l}n \\ k\end{array}\right)^{3}$ the recurrence

$$
(n+2) S(n+2)+(2 n+3) S(n+1)+(n+1) S(n)=0
$$

by creative telescoping. More precisely, we consider the difference field $(\mathbb{E}, \sigma)$ with the rational function field $\mathbb{E}=\mathbb{Q}(n)(k)(b)(h)$ and the automorphism $\sigma$ defined by const ${ }_{\sigma} \mathbb{E}=\mathbb{Q}(n), \sigma(k)=k+1, \sigma(b)=\frac{(n-k)^{3}}{(k+1)^{3}} b$ and $\sigma(h)=h+\frac{1}{k+1}$; note that the shift $S_{k}\left(\begin{array}{l}n \\ k\end{array}\right)^{3}=\frac{(n-k)^{3}}{(k+1)^{3}}\left(\begin{array}{l}n \\ k\end{array}\right)^{3}$ is reflected by the action of $\sigma$ on $b$. Us$\operatorname{ing} S_{n}\left(\begin{array}{l}n \\ k\end{array}\right)^{3}=\frac{(n+1)^{3}}{(n+1-k)^{3}}\left(\begin{array}{l}n \\ k\end{array}\right)^{3}$ we can represent $(f(n, k), f(n+1, k), f(n+2, k))$ in $(\mathbb{E}, \sigma)$ as

$$
\boldsymbol{f}=\left(b(1+h(-6 k+3 n)), \frac{b(1+n)^{3}(1+h(3-6 k+3 n))}{(1-k+n)^{3}}, \frac{b(1+n)^{3}(2+n)^{3}(1+h(6-6 k+3 n))}{\left(2+k^{2}+k(-3-2 n)+3 n+n^{2}\right)^{3}}\right) .
$$

Afterwards we compute the basis $\{(n+1,2 n+3, n+2, g),(0,0,0,1)\}$ with

$$
\begin{aligned}
& g=b k^{2}(1+n)\left(-72+104 k+72 h k-63 k^{2}-102 h k^{2}+18 k^{3}+72 h k^{3}-2 k^{4}\right. \\
& -24 h k^{4}+3 h k^{5}-192 n+208 k n+192 h k n-84 k^{2} n-195 h k^{2} n+12 k^{3} n+90 h k^{3} n \\
& -15 h k^{4} n-186 n^{2}+134 k n^{2}+186 h k n^{2}-27 k^{2} n^{2}-120 h k^{2} n^{2}+27 h k^{3} n^{2}-78 n^{3} \\
& \left.\quad+28 k n^{3}+78 h k n^{3}-24 h k^{2} n^{3}-12 n^{4}+12 h k n^{4}\right) /\left((1-k+n)^{3}(2-k+n)^{3}\right)
\end{aligned}
$$

of the solution space $\mathrm{V}((1,-1), \boldsymbol{f}, \mathbb{E})$; see Example 3.4. Reinterpreting $g$ and $\sigma(g)$ as sequences $g(n, k)$ and $g(n, k+1)$ in terms of $\left(\begin{array}{l}n \\ k\end{array}\right)^{3}$ and $H_{k}$ we get the 
creative telescoping equation

$g(n, k+1)-g(n, k)=(n+1) f(n, k)+(2 n+3) f(n+1, k)+(n+2) f(n+2, k)$

which holds for all $0 \leq k \leq n$. Summing this equation over $k$ from 0 to $n$ gives (3). To this end, any of the algorithms in $[4,42]$ finds the solution $(-1)^{n}$ of (3). By checking initial values we obtain (2).

Finally, we introduce a summation example that is based on problem PLDE with $m>2$.

Example 2.4 In [33, Exp. 3] the following problem has been considered. Given a sequence $T(k)$ for $k \geq 1$ that satisfies the recurrence relation

$$
T(k+2)=\frac{-3\left(3+2 k+\left(2+3 k+k^{2}\right) H_{k}\right)}{(1+k)(2+k) H_{k}} T(k)-\frac{4\left(3+2 k+\left(2+3 k+k^{2}\right) H_{k}\right)}{(2+k)\left(1+(1+k) H_{k}\right)} T(k+1),
$$

find a closed form evaluation of the definite sum $S(n)=\sum_{k=1}^{n}\left(\begin{array}{l}n \\ k\end{array}\right) T(k)$. Here the crucial step was to compute the recurrence relation

$$
\begin{aligned}
12 n(1+n)^{2} S(n)+6 n & (1+n)(3+2 n) S(1+n)+3 n(1+n)(2+n) S(2+n) \\
= & 3\left(6+22 n+13 n^{2}\right) T(1)+2\left(2+7 n+4 n^{2}\right) T(2)
\end{aligned}
$$

by using the algorithms from [33]; note that this approach generalizes the ideas in [11] from the rational case to the $\Pi \Sigma$-field case. Within these computations the essential step consists of solving problem $P L D E$ with $m=3$. More precisely, in [33, Example 9], we needed a non-trivial solution of $\mathrm{V}(\boldsymbol{\alpha}, \boldsymbol{\phi}, \mathbb{E})$ where $(\mathbb{E}, \sigma)$ is defined as in Example 2.3 and $\boldsymbol{\alpha}$ and $\boldsymbol{\phi}$ are given by

$$
\begin{aligned}
\phi & =\left(\frac{b(-k+n)}{1+k}, \frac{b(1+n)}{1+k}, \frac{b(1+n)(2+n)}{(1+k)(1-k+n)}\right) \text { and } \\
\boldsymbol{\alpha} & =\left(\frac{-3\left(11+6 h+12 k+11 h k+3 k^{2}+6 h k^{2}+h k^{3}\right)}{(1+h+h k)\left(6+5 k+k^{2}\right)}, \frac{-4\left(3+2 h+2 k+3 h k+h k^{2}\right)}{(2+k)(1+h+h k)},-1\right) .
\end{aligned}
$$

In Example 3.5 we will show how this non-trivial solution can be computed. To this end, by solving (5) in terms of d'Alembertian solutions, see [8-10,32], one can discover and prove the identity

$S(n)=\frac{27 T(1)+6 T(2)}{18 n}+\frac{1}{18}(3 T(1)+2 T(2))(-2)^{n}\left[H_{n}-\sum_{i=1}^{n} \frac{1}{i(-2)^{i}}\right], \quad n \geq 1$.

We define $\Pi \Sigma$-extensions and $\Pi \Sigma$-fields as follows. A difference field $\left(\mathbb{E}, \sigma^{\prime}\right)$ 
is a difference field extension of $(\mathbb{F}, \sigma)$ if $\mathbb{F}$ is a subfield of $\mathbb{E}$ and $\sigma^{\prime}(g)=\sigma(g)$ for $g \in \mathbb{F}$; note that from now on $\sigma$ and $\sigma^{\prime}$ are not distinguished anymore since they agree on $\mathbb{F}$.

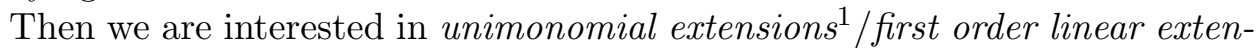
sions $[18,19]$, i.e., difference field extensions $(\mathbb{F}(t), \sigma)$ of $(\mathbb{F}, \sigma)$ where $\mathbb{F}(t)$ is a rational function field, $\sigma$ is defined by $\sigma(t)=\alpha t+\beta$ for some $\alpha \in \mathbb{F}^{*}, \beta \in \mathbb{F}$, and const $_{\sigma} \mathbb{F}(t)=$ const $_{\sigma} \mathbb{F}$.

In particular, we are interested in the following special cases of unimonomial extensions; for more details see $[10,18,19,23]$.

- $\Pi$-extensions, i.e., unimonomial extensions with $\beta=0$.

- $\Sigma^{*}$-extensions, i.e., unimonomial extensions with $\alpha=1$.

- $\Sigma$-extensions, i.e., unimonomial extensions with $\alpha, \beta \in \mathbb{F}^{*}$ where the following two properties hold: (1) there is no $g \in \mathbb{F}$ with $\sigma(g)-\alpha g=\beta$, and (2) if there is an $n \neq 0$ and a $g \in \mathbb{F}^{*}$ with $\alpha^{n}=\frac{\sigma(g)}{g}$ then there is a $g \in \mathbb{F}^{*}$ with $\alpha=\frac{\sigma(g)}{g}$; note that any $\Sigma^{*}$-extension is a $\Sigma$-extension.

- $\Pi \Sigma$-extensions, i.e., $t$ is either a $\Pi$ - or $\Sigma$-extension.

More generally, we consider these extensions in a nested way.

- $\left(\mathbb{F}\left(t_{1}\right) \ldots\left(t_{e}\right), \sigma\right)$ is a (nested) unimonomial (resp. $\left.\Pi \Sigma-/ \Pi-\right)$ extension of $(\mathbb{F}, \sigma)$ if the extension $\left(\mathbb{F}\left(t_{1}, \ldots, t_{i-1}\right)\left(t_{i}\right), \sigma\right)$ of $\left(\mathbb{F}\left(t_{1}, \ldots, t_{i-1}\right), \sigma\right)$ is a unimonomial (resp. $\Pi \Sigma$ - $/ \Pi$-) extension for all $1 \leq i \leq n$; for $i=0$ we define $\mathbb{F}\left(t_{1}\right) \ldots\left(t_{i-1}\right)=\mathbb{F}$.

- $(\mathbb{F}, \sigma)$ is an unimonomial (resp. $\Pi \Sigma$-) field over $\mathbb{K}$ if $\mathbb{F}=\mathbb{K}\left(t_{1}\right) \ldots\left(t_{e}\right),(\mathbb{F}, \sigma)$ is a unimonomial (resp. $\Pi \Sigma$-) extension of $(\mathbb{K}, \sigma)$ and const $_{\sigma} \mathbb{K}=\mathbb{K}$.

Typical examples of $\Pi \Sigma$-extensions and $\Pi \Sigma$-fields are given in Examples 2.2, 2.3 and 2.4 .

We want to emphasize that $\Pi \Sigma$-extensions and $\Pi \Sigma$-fields have two important aspects:

- They contain those unimonomial extensions that are needed to express indefinite nested sums $\left(\Sigma^{*}\right)$ and products $(\Pi)$.

- And they can be constructed in an automatic fashion if the constant field $\mathbb{K}$ is $\sigma$-computable, i.e., the following three properties hold. (1) For any $k \in \mathbb{K}$ one can decide if $k \in \mathbb{Z},(\mathbf{2})$ there is an algorithm that can factorize multivariate polynomials in $\mathbb{K}\left[t_{1}, \ldots, t_{e}\right]$, and (3) there is an algorithm that can compute a basis of the submodule $\left\{\left(n_{1}, \ldots, n_{k}\right) \in \mathbb{Z}^{k} \mid c_{1}^{n_{1}} \cdots c_{k}^{n_{k}}=1\right\}$ of $\mathbb{Z}^{k}$ over $\mathbb{Z}$ for any $\left(c_{1}, \ldots, c_{k}\right) \in \mathbb{K}^{k}$. E.g., any rational function field

\footnotetext{
${ }^{1}$ Note that in [23] unimonomial extensions are defined in a more general context that covers also differential extensions. Moreover our special case restricts to those extensions with $\operatorname{const}_{\sigma} \mathbb{F}(t)=$ const $_{\sigma} \mathbb{F}$.
} 
$\mathbb{K}=\mathbb{A}\left(x_{1}, \ldots, x_{r}\right)$ over an algebraic number field $\mathbb{A}$ is $\sigma$-computable; see [20].

For further details concerning the construction of $\Pi \Sigma$-fields we refer to $[18,26]$. Refined constructions of $\Pi \Sigma$-fields are given in $[20,27,28]$.

Finally, we introduce some additional notation. Let $\mathbb{F}$ be a field and $\boldsymbol{f}=$ $\left(f_{1}, \ldots, f_{n}\right) \in \mathbb{F}^{n}$. If $c \in \mathbb{F}$ we define $c \boldsymbol{f}:=\left(c f_{1}, \ldots, c f_{n}\right)$; if $\boldsymbol{c} \in \mathbb{F}^{n}$, we define the vector product $\boldsymbol{c f}:=\sum_{i=1}^{n} c_{i} f_{i}$. With $\boldsymbol{M} \boldsymbol{f}^{t} \in \mathbb{F}^{m}$ we denote the usual multiplication of a matrix $\boldsymbol{M} \in \mathbb{F}^{m \times n}$ with the transposed vector $\boldsymbol{f}^{t}$; if it is clear from the context, we also write $\boldsymbol{M} \boldsymbol{f}$. For a function $\sigma: \mathbb{F} \rightarrow \mathbb{F}$ and $g \in \mathbb{F}$ we define $\sigma(\boldsymbol{f}):=\left(\sigma\left(f_{1}\right), \ldots, \sigma\left(f_{n}\right)\right) \in \mathbb{F}^{n}$ and $\sigma_{\boldsymbol{f}} g:=f_{1} \sigma^{n-1}(g)+\cdots+f_{n} g \in$ $\mathbb{F}$. $\mathbf{I d}_{n}$ stands for the identity matrix and $\mathbf{0}_{n}$ stands for the zero-vector of length $n$.

Let $\mathbb{K}$ be a subfield of $\mathbb{F}$. Then we define the subspace Nullspace $_{\mathbb{K}}(\boldsymbol{f})$ of $\mathbb{K}^{n}$ given by

$$
\text { Nullspace }_{\mathbb{K}}(\boldsymbol{f}):=\left\{\boldsymbol{k} \in \mathbb{K}^{n} \mid \boldsymbol{f} \boldsymbol{k}=0\right\} .
$$

Moreover, let $\mathbb{F}[t]$ be a polynomial ring. We introduce

$$
t^{b} \mathbb{F}:=\left\{t^{b} f \mid f \in \mathbb{F}\right\} \quad \text { and } \quad \mathbb{F}[t]_{b}:=\{f \in \mathbb{F}[t] \mid \operatorname{deg}(f) \leq b\}
$$

for $b \in \mathbb{N}_{0}$, and $\mathbb{F}[t]_{-1}:=\{0\}$. Moreover, we define $\|f\|:=\operatorname{deg} f$ for $f \in \mathbb{F}[t]^{*}$, $\|0\|:=-1$, and $\|\boldsymbol{f}\|:=\max _{1 \leq i \leq n}\left\|f_{i}\right\|$ for $\boldsymbol{f}=\left(f_{1}, \ldots, f_{n}\right) \in \mathbb{F}[t]^{n}$. $[p]_{l}$ gives the $l$-th coefficient of $p \in \mathbb{F}[t]$. Furthermore, we denote

$$
\mathbb{F}(t)^{(\text {frac })}:=\left\{\frac{p}{q} \mid p, q \in \mathbb{F}[t] \text { and }\|p\|<\|q\|\right\},
$$

i.e., $\mathbb{F}(t)=\mathbb{F}[t] \oplus \mathbb{F}(t)^{(\text {frac })}$ where $\mathbb{F}[t], \mathbb{F}(t)^{(\text {frac })}$ are considered as subspaces of $\mathbb{F}(t)$ over $\mathbb{K}$.

Let $(\mathbb{F}, \sigma)$ be a difference field and $f \in \mathbb{F}^{*}$. Then we define the $\sigma$-factorial $f_{(k)}$ for a non-negative integer by $\prod_{i=0}^{k-1} \sigma^{i}(f)$. The proof of the following lemma is left to the reader.

Lemma 2.5 Let $(\mathbb{F}(t), \sigma)$ be a unimonomial extension of $(\mathbb{F}, \sigma)$ with $\sigma(t)=$ $\alpha t+\beta$. Then for any non-negative integer $k, \sigma^{k}(t)=\alpha_{(k)} t+b$ for some $b \in \mathbb{F}$.

\section{The reduction strategy}

Given a unimonomial extension $(\mathbb{F}(t), \sigma)$ of $(\mathbb{F}, \sigma)$, we try to solve problem $P L D E$ in the following way. First we compute a common denominator of all the possible solutions in $\mathbb{F}(t)$ and afterwards we compute the "numerator" 
of the solutions over this common denominator. More precisely, we propose a reduction strategy that can be summarized in

THEOREM 3.1 Let $(\mathbb{F}(t), \sigma)$ be a unimonomial extension of $(\mathbb{F}, \sigma)$ with $\mathbb{K}:=$ const $_{\sigma} \mathbb{F}$. Then one can solve problem PLDE in $(\mathbb{F}(t), \sigma)$ if one can solve problems DenB and DegB, see Subsection 3.2, and problems PLDE and NS in $(\mathbb{F}, \sigma)$, see Subsection 3.3.

Subsequently, let $(\mathbb{F}(t), \sigma)$ be a unimonomial extension of $(\mathbb{F}, \sigma)$ with $\sigma(t)=$ $\alpha t+\beta, \mathbb{K}=$ const $_{\sigma} \mathbb{F}$, and let $\mathbf{0} \neq \boldsymbol{a}=\left(a_{1}, \ldots, a_{m}\right) \in \mathbb{F}(t)^{m}$ and $\boldsymbol{f} \in \mathbb{F}(t)^{n}$.

\subsection{Simplifications and shortcuts.}

In a first step we try to decrease the order of the parameterized linear difference equation, i.e., we try to decrease $m$. Moreover, we consider two shortcuts which allow us to compute a basis in one stroke.

Simplification I. If $a_{1} a_{m}=0$, we can reduce the order as follows. If $a_{1} \neq 0$, set $l:=1$, otherwise take that $l$ with $0=a_{1}=\cdots=a_{l-1} \neq a_{l}$. Similarly, if $a_{m} \neq 0$, set $k:=m$, otherwise take that $k$ with $a_{k} \neq a_{k+1}=\cdots=a_{m}=0$. Then we have

$$
\sigma_{\boldsymbol{a}} g=\boldsymbol{c} \boldsymbol{f} \quad \Leftrightarrow \quad \sigma^{k-m}\left(a_{l}\right) \sigma^{k-l}(g)+\cdots+\sigma^{k-m}\left(a_{k}\right) g=\boldsymbol{c} \sigma^{k-m}(\boldsymbol{f})
$$

where $\sigma^{k-m}\left(a_{l}\right) \neq 0 \neq \sigma^{k-m}\left(a_{k}\right)$. Therefore define $\boldsymbol{a}^{\prime} \in \mathbb{F}(t)^{k-l+1}$ and $\boldsymbol{f}^{\prime} \in$ $\mathbb{F}(t)^{n}$ by

$$
\boldsymbol{a}^{\prime}:=\left(\sigma^{k-m}\left(a_{l}\right), \ldots, \sigma^{k-m}\left(a_{k}\right)\right) \quad \text { and } \quad \boldsymbol{f}^{\prime}:=\sigma^{k-m}(\boldsymbol{f}),
$$

and find a basis of $\mathrm{V}\left(\boldsymbol{a}^{\prime}, \boldsymbol{f}^{\prime}, \mathbb{F}(t)\right)$, say $\left\{\left(c_{i 1}, \ldots, c_{i n}, g_{i}\right)\right\}_{1 \leq i \leq r} \subseteq \mathbb{K}^{n} \times \mathbb{F}(t)$. Then $\left\{\left(c_{i 1}, \ldots, c_{i n}, \sigma^{m-k}\left(g_{i}\right)\right)\right\}_{1 \leq i \leq r} \subseteq \mathbb{K}^{n} \times \mathbb{F}(t)$ is a basis of $\mathrm{V}(\boldsymbol{a}, \boldsymbol{f}, \mathbb{F}(t))$.

Hence we may suppose that $\boldsymbol{a}=\left(a_{1}, \ldots, a_{m}\right) \in \mathbb{F}(t)^{m}$ with $a_{1} a_{m} \neq 0$.

Shortcut I. If $m=1$, we can produce a basis as follows. Define $\boldsymbol{g}:=\frac{\boldsymbol{f}}{a_{1}}$. Then it follows with $\boldsymbol{g}=\left(g_{1}, \ldots, g_{r}\right)$ and the $i$-th unit vector $(0 \ldots, 1, \ldots, 0) \in \mathbb{K}^{n}$ that $\left\{\left(0 \ldots, 1, \ldots, 0, g_{i}\right)\right\}_{1 \leq i \leq r} \subseteq \mathbb{K}^{n} \times \mathbb{F}(t)$ is a basis of $\mathrm{V}(\boldsymbol{a}, \boldsymbol{f}, \mathbb{F}(t))$.

Therefore we may suppose $\boldsymbol{a}=\left(a_{1}, \ldots, a_{m}\right) \in \mathbb{F}(t)^{m}$ with $a_{1} a_{m} \neq 0, m>1$.

Simplification II. If $a_{i}=0$ for all $1<i<m$ one is able to reduce the problem further. To accomplish this task, we use the fact that if $(\mathbb{F}(t), \sigma)$ is a unimonomial extension of $(\mathbb{F}, \sigma)$, then also $\left(\mathbb{F}(t), \sigma^{m-1}\right)$ is a unimonomial extension of $\left(\mathbb{F}, \sigma^{m-1}\right)$ and that $\mathrm{V}(\boldsymbol{a}, \boldsymbol{f}, \mathbb{F}(t))$ in $(\mathbb{F}(t), \sigma)$ is equal to $\mathbb{V}:=$ $\mathrm{V}\left(\left(a_{1}, a_{m}\right), \boldsymbol{f}, \mathbb{F}(t)\right)$ in $\left(\mathbb{F}(t), \sigma^{m-1}\right)$.

Remark. Suppose that $(\mathbb{F}(t), \sigma)$ is a $\Pi \Sigma$-field over a $\sigma$-computable $\mathbb{K}$. Then 
by [19, Thm: page 314$]\left(\mathbb{F}(t), \sigma^{m-1}\right)$ is a $\Pi \Sigma$-field over $\mathbb{K}$, i.e., a basis of $\mathbb{V}$ can be computed by Theorem 4.7 .

Clearing denominators and cancelling common factors. Compute $\boldsymbol{a}^{\prime}=\left(a_{1}^{\prime}, \ldots, a_{m}^{\prime}\right) \in \mathbb{F}[t]^{m}$ and $\boldsymbol{f}^{\prime}=\left(f_{1}^{\prime}, \ldots, f_{n}^{\prime}\right) \in \mathbb{F}[t]^{n}$ such that $\operatorname{gcd}_{\mathbb{F}[t]}\left(f_{1}^{\prime}, \ldots, f_{n}^{\prime}, a_{1}^{\prime}, \ldots, a_{m}^{\prime}\right)=1$ and $\boldsymbol{a}^{\prime}=\boldsymbol{a} q, \boldsymbol{f}^{\prime}=\boldsymbol{f} q$ for some $q \in \mathbb{F}(t)^{*}$. Then $\mathrm{V}(\boldsymbol{a}, \boldsymbol{f}, \mathbb{F}(t))=\mathrm{V}\left(\boldsymbol{a}^{\prime}, \boldsymbol{f}^{\prime}, \mathbb{F}(t)\right)$.

Thus we may suppose that the entries in $\mathbf{0} \neq \boldsymbol{a} \in \mathbb{F}[t]^{m}, \boldsymbol{f} \in \mathbb{F}[t]^{n}$ have no common factors.

Shortcut II. We have $\mathrm{V}(\boldsymbol{a}, \boldsymbol{f}, \mathbb{K})=$ Nullspace $_{\mathbb{K}}(\boldsymbol{h})$ by taking the vector $\boldsymbol{h}:=$ $\left(f_{1}, \ldots, f_{n},-\sum_{i=1}^{m} a_{i}\right)$. Hence this special case can be reduced to problem $N S$. NS: Nullspace

- Given a rational function field $\mathbb{F}(t)$ with subfield $\mathbb{K}$ and $\boldsymbol{f} \in \mathbb{F}[t]^{n}$.

- Find a basis of Nullspace $\mathbb{K}_{\mathbb{K}}(\boldsymbol{f})=\left\{\boldsymbol{k} \in \mathbb{K}^{n} \mid \boldsymbol{f} \boldsymbol{k}=0\right\}$ over $\mathbb{K}$.

It is easy to see that one can solve problem $N S$ with linear algebra methods if $(\mathbb{F}, \sigma)$ is a unimonomial field over a $\sigma$-computable $\mathbb{K}$; see [39, Lemma 5.3]. Hence we get

Lemma 3.2 Let $(\mathbb{F}(t), \sigma)$ be a unimonomial field (resp. $\Pi \Sigma$-field) over a $\sigma$ computable $\mathbb{K}$. Then one can solve problem NS and problem PLDE in $(\mathbb{K}, \sigma)$ with linear algebra methods.

\subsection{Bounds for the solution space}

In the second reduction step one tries to solve the problems $\operatorname{Den} B$ and $\operatorname{Deg} B$ given below. Note that the solutions of these problems are not subject of the present paper; see Remark 4.8.

DenB: Denominator Bounding.

- Given a unimonomial extension $(\mathbb{F}(t), \sigma)$ of $(\mathbb{F}, \sigma), \mathbf{0} \neq \boldsymbol{a} \in \mathbb{F}[t]^{m}$ and $\boldsymbol{f} \in \mathbb{F}[t]^{n}$.

- Find a denominator bound of $\mathrm{V}(\boldsymbol{a}, \boldsymbol{f}, \mathbb{F}(t))$, i.e., a polynomial $d \in \mathbb{F}[t]^{*}$ that fulfills

$$
\forall\left(c_{1}, \ldots, c_{n}, g\right) \in \mathrm{V}(\boldsymbol{a}, \boldsymbol{f}, \mathbb{F}(t)): d g \in \mathbb{F}[t] .
$$

Since $\mathrm{V}(\boldsymbol{a}, \boldsymbol{f}, \mathbb{F}(t))$ is finite-dimensional over $\mathbb{K}$, a denominator bound exists. Suppose that we are given such a $d$ and define

$$
\boldsymbol{a}^{\prime}:=\left(\frac{a_{1}}{\sigma^{m-1}(d)}, \frac{a_{2}}{\sigma^{m-2}(d)}, \ldots, \frac{a_{m}}{d}\right) .
$$

Note that $\left\{\left(c_{i 1}, \ldots, c_{i n}, g_{i}\right)\right\}_{1 \leq i \leq r}$ is a basis of $\mathrm{V}\left(\boldsymbol{a}^{\prime}, \boldsymbol{f}, \mathbb{F}[t]\right)$ if and only if $\left\{\left(c_{i 1}, \ldots, c_{i n}, \frac{g_{i}}{d}\right)\right\}_{1 \leq i \leq r}$ is a basis of $\mathrm{V}(\boldsymbol{a}, \boldsymbol{f}, \mathbb{F}(t))$. Hence, given a denominator bound $d$ of $\mathrm{V}(\boldsymbol{a}, \boldsymbol{f}, \mathbb{F}(t))$, we can reduce the problem of searching for a basis of 
$\mathrm{V}(\boldsymbol{a}, \boldsymbol{f}, \mathbb{F}(t))$ to looking for a basis of $\mathrm{V}\left(\boldsymbol{a}^{\prime}, \boldsymbol{f}, \mathbb{F}[t]\right)$. By clearing denominators and cancelling common factors in $\boldsymbol{a}^{\prime}$ and $\boldsymbol{f}$, as above, we may also suppose that $\boldsymbol{a}=\left(a_{1}, \ldots, a_{m}\right) \in \mathbb{F}[t]^{m}$ with $a_{1} a_{m} \neq 0, m>1$, and $\boldsymbol{f} \in \mathbb{F}[t]^{n}$.

The next reduction step consists of bounding the polynomial degrees in $\mathrm{V}(\boldsymbol{a}, \boldsymbol{f}, \mathbb{F}[t])$.

\section{DegB: Degree Bounding}

- Given a unimonomial extension $(\mathbb{F}(t), \sigma)$ of $(\mathbb{F}, \sigma), \mathbf{0} \neq \boldsymbol{a} \in \mathbb{F}[t]^{m}$ and $\boldsymbol{f} \in \mathbb{F}[t]^{n}$.

- Find a degree bound $b \in \mathbb{N}_{0} \cup\{-1\}$, i.e., $\mathrm{V}\left(\boldsymbol{a}, \boldsymbol{f}, \mathbb{F}[t]_{b}\right)=\mathrm{V}(\boldsymbol{a}, \boldsymbol{f}, \mathbb{F}[t])$.

Besides this we will assume that a degree bound satisfies always the inequality

$$
b \geq\|\boldsymbol{f}\|-\|\boldsymbol{a}\| .
$$

Again, since $\mathrm{V}(\boldsymbol{a}, \boldsymbol{f}, \mathbb{F}[t])$ is finite-dimensional over $\mathbb{K}$, a degree bound must exist.

Example 3.3 (Cont. Exp. 2.2) By [25, Cor. 1] we can take the denominator bound 1 for $\mathrm{V}((1,-1),(h), \mathbb{Q}(k)(h))$, and by $[26$, Cor. 6$]$ we can take the degree bound 2 for $\mathrm{V}((1,-1),(h), \mathbb{Q}(k)[h])$. Hence we have to compute a basis of $\mathrm{V}((1,-1),(h), \mathbb{Q}(k)(h))=\mathrm{V}\left((1,-1),(h), \mathbb{Q}(k)[h]_{2}\right)$; see Example 3.7.

Example 3.4 (Cont. Exp. 2.3) Denote $\mathbb{F}:=\mathbb{Q}(n)(k)(b)$. By [25, Cor. 1] a denominator bound of $\mathrm{V}((1,-1), \boldsymbol{f}, \mathbb{F}(h))$ is 1 and by [26, Cor. 6] a degree bound of $\mathrm{V}((1,-1), \boldsymbol{f}, \mathbb{F}[h])$ is 2 . Finally, in Example 3.8 we will compute a basis of $\mathrm{V}((1,-1), \boldsymbol{f}, \mathbb{F}(h))=\mathrm{V}\left((1,-1), \boldsymbol{f}, \mathbb{F}[h]_{2}\right)$.

Example 3.5 (Cont. Exp. 2.4) Denote $\mathbb{F}:=\mathbb{Q}(n)(k)(b)$. By [25, Alg. 3] we compute the denominator bound $d=(k+1) h+1$ of $\mathrm{V}(\boldsymbol{\alpha}, \boldsymbol{\phi}, \mathbb{F}(h))$. After adapting $^{1} \boldsymbol{\alpha}$ and $\phi$ to

$$
\begin{aligned}
& \boldsymbol{a}=\left(-3(1+k)^{2}(2+k)(-1+k-n),\right. \\
& \left.-4(1+k)^{2}(3+k)(-1+k-n),(-1-k)(2+k)(3+k)(-1+k-n)\right), \\
& \boldsymbol{f}=\left(b(3+k)\left(2+k+h\left(2+3 k+k^{2}\right)\right)(k-n)(1-k+n),\right. \\
& b(3+k)\left(2+k+h\left(2+3 k+k^{2}\right)\right)(-1-n)(1-k+n), \\
& \left.b(3+k)\left(2+k+h\left(2+3 k+k^{2}\right)\right)(-1-n)(2+n)\right)
\end{aligned}
$$

by following (8), the task is to find a non-trivial solution of $\mathrm{V}(\boldsymbol{a}, \boldsymbol{f}, \mathbb{F}[h])$. By checking that there is no $g \in \mathbb{F}$ with $\sigma_{\boldsymbol{a}} g=0$ we can apply [25, Prop. 2]

\footnotetext{
${ }^{1}$ In this example we cancelled also the units in the denominators.
} 
and obtain the degree bound 1 for $\mathrm{V}(\boldsymbol{a}, \boldsymbol{f}, \mathbb{F}[h])$; note that this check can be done again by our algorithms. Given this information, we compute for $\mathrm{V}\left(\boldsymbol{a}, \boldsymbol{f}, \mathbb{F}[h]_{1}\right)$ the solution $\mathcal{B}_{1}=\left\{\left(c_{1}, c_{2}, c_{3}, g\right)\right\}$ where $c_{1}=4 n^{2}(1+n)^{2}, c_{2}=$ $2 n^{2}(1+n)(3+2 n), c_{3}=n^{2}(1+n)(2+n)$ and

$$
\begin{aligned}
g= & -b(1+k)\left(2 k^{2}(1+n)^{2}(1+h n)+n(1+n)(2+3 n(2+n))\right. \\
& -k(2+n(8+n(13+6 n)+h(1+n)(2+3 n(2+n))))) /(-1+k-n) ;
\end{aligned}
$$

see Example 3.9. This gives one particular solution $\left(c_{1}, c_{2}, c_{3}, \frac{g}{(k+1) h+1}\right)$ for $\mathrm{V}(\boldsymbol{\alpha}, \boldsymbol{\phi}, \mathbb{F}(h))$.

By (9) we have $\boldsymbol{f} \in \mathbb{F}[t]_{\|\boldsymbol{a}\|+b}^{n}$. Hence we can proceed as follows by taking $\delta:=b$.

\subsection{Incremental reduction or polynomial degree reduction}

We are interested in the following problem. Given $\delta \in \mathbb{N}_{0} \cup\{-1\}, \mathbf{0} \neq \boldsymbol{a} \in$ $\mathbb{F}[t]^{m}$ with $l:=\|\boldsymbol{a}\|$ and $\boldsymbol{f} \in \mathbb{F}[t]_{\delta+l}^{n}$, find a basis $\mathcal{B}_{\delta}$ of $\mathrm{V}\left(\boldsymbol{a}, \boldsymbol{f}, \mathbb{F}[t]_{\delta}\right)$. In order to accomplish this task, we shall develop a reduction strategy that can be summarized as follows.

THEOREM 3.6 Let $(\mathbb{F}(t), \sigma)$ be a unimonomial extension of $(\mathbb{F}, \sigma)$ with $\mathbb{K}:=$ const $_{\sigma} \mathbb{F}, \mathbf{0} \neq \boldsymbol{a} \in \mathbb{F}[t]^{m}$ with $l:=\|\boldsymbol{a}\|$, and $\boldsymbol{f} \in \mathbb{F}[t]_{\delta+l}^{n}$ for some $\delta \in \mathbb{N}_{0} \cup\{-1\}$. Then one can find a basis of $\mathrm{V}\left(\boldsymbol{a}, \boldsymbol{f}, \mathbb{F}[t]_{\delta}\right)$ if one can solve problem $N S$, and one can solve problem PLDE in $(\mathbb{F}, \sigma)$.

This reduction, a generalization of [18, Thm. 12], can be considered as the inner core of our method. Observe that together with the previous subsections this result will show our main result stated in Theorem 3.1.

Subsequently, let $\boldsymbol{a}, \boldsymbol{f}_{\delta}:=\boldsymbol{f}, l$ and $\delta$ as posed in Theorem 3.6. First we consider the base case of our reduction and a shortcut.

Base case: $\delta=-1$. In this case we have $\mathrm{V}(\boldsymbol{a}, \boldsymbol{f},\{0\})=$ Nullspace $_{\mathbb{K}}(\boldsymbol{f}) \times\{0\}$, i.e., we have to solve problem $N S$.

Shortcut: $\boldsymbol{a} \in \mathbb{F}^{m}$ and $\delta=0$. Then $\mathbb{F}[t]_{\delta+l}=\mathbb{F}$ and $\mathbb{F}[t]_{\delta}=\mathbb{F}$, i.e., we can compute a basis of $\mathrm{V}\left(\boldsymbol{a}, \boldsymbol{f}, \mathbb{F}[t]_{\delta}\right)$ under the assumption that one can solve problem $P L D E$ in $(\mathbb{F}, \sigma)$.

If $\delta \geq 0$ we can proceed as follows. First we find the candidates of the leading coefficients $g_{\delta} \in \mathbb{F}$ for the solutions $\left(c_{1}, \ldots, c_{n}, g\right) \in \mathrm{V}\left(\boldsymbol{a}, \boldsymbol{f}_{\delta}, \mathbb{F}[t]_{\delta}\right)$ with $g=\sum_{i=0}^{\delta} g_{i} t^{i}$, plugging back its solution space and go on recursively to derive the candidates of the missing coefficients $g_{i} \in \mathbb{F}$.

Example 3.7 (Cont. Exp. 2.2) By Example 3.3 we have to compute a basis of the solution space $\mathbb{V}:=\mathrm{V}\left((1,-1),(h), \mathbb{Q}(k)[h]_{2}\right)$. Since $(1,0) \in \mathbb{V}$ it remains 


$$
\begin{aligned}
& \boldsymbol{f}_{\delta}:=\boldsymbol{f}, \lambda_{\delta}:=n \\
& \mathrm{~V}\left(\boldsymbol{a}, \boldsymbol{f}_{\delta}, \mathbb{F}[t]_{\delta}\right) \quad(i) \\
& \text { (iii) } \uparrow \quad \\
& \mathrm{V}\left(\tilde{\boldsymbol{a}}_{\delta}, \tilde{\boldsymbol{f}}_{\delta}, \mathbb{F}\right) \quad \tilde{\boldsymbol{a}}_{\delta} \in \mathbb{F}^{m}, \tilde{\boldsymbol{f}}_{\delta} \in \mathbb{F}^{\lambda_{\delta}} \\
& \boldsymbol{f}_{\delta-1} \in \mathbb{F}[t]_{l+d-1}^{\lambda_{\delta-1}} \\
& \mathrm{~V}\left(\boldsymbol{a}, \boldsymbol{f}_{\delta-1}, \mathbb{F}[t]_{\delta-1}\right) \\
& \text { (ii) } \\
& \boldsymbol{f}_{0} \in \mathbb{F}[t]_{l}^{\lambda_{0}} \\
& \boldsymbol{f}_{-1} \in \mathbb{F}[t]_{l-1}^{\lambda_{-1}}
\end{aligned}
$$
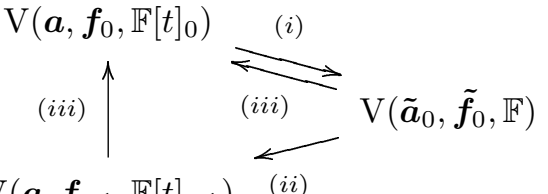

Figure 1. Incremental reduction

to look for a $g=g_{2} h^{2}+g_{1} h+g_{0} \in \mathbb{Q}(k)[h]_{2}$ with $\sigma(g)-g=h$, i.e.,

$$
\left[\sigma\left(g_{2}\right)\left(h+\frac{1}{k+1}\right)^{2}+\sigma\left(g_{1} h+g_{0}\right)\right]-\left[g_{2} h^{2}+g_{1} h+g_{0}\right]=h .
$$

By comparing the leading coefficients in (10) we obtain the constraint $\sigma\left(g_{2}\right)-$ $g_{2}=0$, i.e., $g_{2}=c \in$ const $_{\sigma} \mathbb{Q}(k)=\mathbb{Q}$. Plugging this result back into (10) gives

$$
\sigma\left(g_{1} t+g_{0}\right)-\left(g_{1} t+g_{0}\right)=t-c \frac{2 t(k+1)+1}{(k+1)^{2}},
$$

where the highest degree has been reduced by one. Again, by comparing the leading coefficients in (11) we get the condition $\sigma\left(g_{1}\right)-g_{1}=1-c \frac{2}{k+1}$. Solving this problem in $(\mathbb{Q}(k), \sigma)$ gives $c=0$ and $g_{1}=k+d$ with $d \in \mathbb{Q}$. Plugging back this solution into (11), we obtain $\sigma\left(g_{0}\right)-g_{0}=-1-d \frac{1}{k+1}$. This can be solved in $(\mathbb{Q}(k), \sigma)$ with $g_{0}=-k$ and $d=0$. Summarizing, $g=k t-k$ is a solution of $\sigma(g)-g=h$, and $\{(0,1),(1, g)\}$ is a basis of $\mathrm{V}((1,-1),(h), \mathbb{Q}(k)(h))$.

The reduction idea is graphically illustrated in Figure 1, which has to be read as follows. The problem of finding a basis $\mathcal{B}_{\delta}$ of $\mathrm{V}\left(\boldsymbol{a}, \boldsymbol{f}_{\delta}, \mathbb{F}[t]_{\delta}\right)$ is reduced to (i) searching for the possible leading coefficients, i.e., to searching for a basis $\tilde{\mathcal{B}}_{\delta}$ of $\mathrm{V}\left(\tilde{\boldsymbol{a}}_{\delta}, \tilde{\boldsymbol{f}}_{\delta}, \mathbb{F}\right)$ with (12), and (ii) finding the polynomials with the remaining coefficients, i.e., finding a basis $\mathcal{B}_{\delta-1}$ of $\mathrm{V}\left(\boldsymbol{a}, \boldsymbol{f}_{\delta-1}, \mathbb{F}[t]_{\delta-1}\right)$ with (15). Then (iii), a basis $\mathcal{B}_{\delta}$ of $\mathrm{V}\left(\boldsymbol{a}, \boldsymbol{f}_{\delta}, \mathbb{F}[t]_{\delta}\right)$ can be reconstructed by the two bases $\tilde{\mathcal{B}}_{\delta}$ and $\mathcal{B}_{\delta-1}$ of the corresponding subproblems; see (17). 
Subsequently, we explain our reduction; for a rigorous proof see [39]. Define

$$
\tilde{\boldsymbol{a}}_{\delta}=\left(\tilde{a}_{1}, \ldots, \tilde{a}_{m}\right):=\left(\alpha_{(m-1)}^{\delta}\left[a_{1}\right]_{l}, \ldots, \alpha_{(0)}^{\delta}\left[a_{m}\right]_{l}\right), \quad \tilde{\boldsymbol{f}}_{\delta}:=\left(\left[f_{1}\right]_{\delta+l}, \ldots,\left[f_{n}\right]_{\delta+l}\right)
$$

where $\mathbf{0} \neq \tilde{\boldsymbol{a}}_{\delta} \in \mathbb{F}^{m}$ and $\tilde{\boldsymbol{f}}_{\delta} \in \mathbb{F}^{n}$. Then there is the following crucial observation for a solution $\boldsymbol{c} \in \mathbb{K}^{n}$ and $g=\sum_{i=0}^{\delta} g_{i} t^{i} \in \mathbb{F}[t]_{\delta}$ of $\mathrm{V}\left(\boldsymbol{a}, \boldsymbol{f}_{\delta}, \mathbb{F}[t]_{\delta}\right)$ : Since $t$ is transcendental over $\mathbb{F}$, it follows by leading coefficient comparison and Lemma 2.5 that

$$
\sigma_{\tilde{\boldsymbol{a}}} g_{\delta}=\boldsymbol{c} \tilde{\boldsymbol{f}}_{\delta}
$$

i.e., $\left(c_{1}, \ldots, c_{n}, g_{\delta}\right) \in \mathrm{V}\left(\tilde{\boldsymbol{a}}_{\delta}, \tilde{\boldsymbol{f}}_{\delta}, \mathbb{F}\right)$; see [39, Lemma 6.1]. Therefore, the right linear combinations of a basis of $\mathrm{V}\left(\tilde{\boldsymbol{a}}_{\delta}, \tilde{\boldsymbol{f}}_{\delta}, \mathbb{F}\right)$ enable one to construct partially the solutions $\left(c_{1}, \ldots, c_{n}, g\right) \in \mathrm{V}\left(\boldsymbol{a}, \boldsymbol{f}_{\delta}, \mathbb{F}[t]_{\delta}\right)$, namely $\left(c_{1}, \ldots, c_{n}\right) \in \mathbb{K}^{n}$ with the $\delta$-th coefficient $g_{\delta}$ in $g \in \mathbb{F}[t]_{\delta}$. So, the basic idea is to find first a basis $\tilde{\mathcal{B}}_{\delta}$ of $\mathrm{V}\left(\tilde{\boldsymbol{a}}_{\delta}, \tilde{\boldsymbol{f}}_{\delta}, \mathbb{F}\right)$.

- CASE I: $\tilde{\mathcal{B}}_{\delta}=\{\}$. Thus $\boldsymbol{c}=\mathbf{0}$ and $g \in \mathbb{F}[t]_{\delta-1}$ are the only candidates for $\sigma_{\boldsymbol{a}} g=\boldsymbol{c} \boldsymbol{f}$. Hence, take a basis $\mathcal{B}_{\delta-1}$ of $\mathrm{V}\left(\boldsymbol{a}, \boldsymbol{f}_{\delta-1}, \mathbb{F}[t]_{\delta-1}\right)$ with $\boldsymbol{f}_{\delta-1}:=(0)$ and extract a basis $H \subseteq \mathbb{F}[t]_{\delta-1}^{*}$ for the vector space

$$
\left\{h \in \mathbb{F}[t]_{\delta-1} \mid \sigma_{\boldsymbol{a}} h=0\right\} .
$$

If $H=\left\{g_{1}, \ldots, g_{\mu}\right\} \neq\{\}$, a basis of $\mathrm{V}\left(\boldsymbol{a}, \boldsymbol{f}_{\delta}, \mathbb{F}[t]_{\delta}\right)$ is $\left(0, \ldots, 0, g_{i}\right)_{1 \leq i \leq \mu} \subseteq$ $\mathbb{K}^{n} \times \mathbb{F}[t]_{\delta-1}$. Otherwise, $\mathrm{V}\left(\boldsymbol{a}, \boldsymbol{f}_{\delta}, \mathbb{F}[t]_{\delta}\right)=\left\{\mathbf{0}_{n+1}\right\} ;$ for further details see the proof of [39, Cor. 6.1].

- CASE II: $\tilde{\mathcal{B}}_{\delta} \neq\{\}$, say $\tilde{\mathcal{B}}_{\delta}=\left\{\left(c_{i 1}, \ldots, c_{i n}, w_{i}\right)\right\}_{1 \leq i \leq \lambda}$. Then define

$$
\boldsymbol{C}:=\left(c_{i j}\right) \in \mathbb{K}^{\lambda \times n} \quad \text { and } \quad \boldsymbol{g}:=\left(w_{1} t^{\delta}, \ldots, w_{\lambda} t^{\delta}\right)
$$

with $\boldsymbol{g} \in t^{\delta} \mathbb{F}^{\lambda}$ and consider

$$
\boldsymbol{f}_{\delta-1}:=\boldsymbol{C} \boldsymbol{f}_{\delta}-\sigma_{\boldsymbol{a}} \boldsymbol{g}
$$

By construction it follows that $\boldsymbol{f}_{\delta-1} \in \mathbb{F}[t]_{\delta+l-1}^{\lambda}$. Now we proceed as follows. We try to determine exactly those $h \in \mathbb{F}[t]_{\delta-1}$ and $\boldsymbol{d} \in \mathbb{K}^{\lambda}$ that fulfill

$$
\sigma_{\boldsymbol{a}}(h+\boldsymbol{d g})=\boldsymbol{d} \boldsymbol{C} \boldsymbol{f}_{\delta}, \quad \text { i.e., } \quad \sigma_{\boldsymbol{a}} h=\boldsymbol{d} \boldsymbol{f}_{\delta-1} .
$$

For this task, we take a basis $\mathcal{B}_{\delta-1}$ of $\mathrm{V}\left(\boldsymbol{a}, \boldsymbol{f}_{\delta-1}, \mathbb{F}[t]_{\delta-1}\right)$.

- CASE II.i: $\mathcal{B}_{\delta-1}=\{\}$. Then $\operatorname{V}\left(\boldsymbol{a}, \boldsymbol{f}_{\delta}, \mathbb{F}[t]_{\delta}\right)=\left\{\mathbf{0}_{n+1}\right\}$. 
- CASE II.ii: $\mathcal{B}_{\delta-1} \neq\{\}$, say $\mathcal{B}_{\delta-1}=\left\{\left(d_{i 1}, \ldots, d_{i \lambda}, h_{i}\right)\right\}_{1 \leq i \leq \mu}$. Then define $\boldsymbol{D}:=\left(d_{i j}\right) \in \mathbb{K}^{\mu \times \lambda}$ and $\boldsymbol{h}:=\left(h_{1}, \ldots, h_{\mu}\right) \in \mathbb{F}[]_{\delta-1}^{\mu}$. It is important to observe that

$$
\sigma_{\boldsymbol{a}}(\boldsymbol{h}+\boldsymbol{D g})=\boldsymbol{D} \boldsymbol{C} \boldsymbol{f}_{\delta}
$$

Now define $\kappa_{i j} \in \mathbb{K}$ and $p_{i} \in \mathbb{F}[t]_{\delta}^{\mu}$ with

$$
\left(\begin{array}{ccc}
\kappa_{11} & \ldots & \kappa_{1 n} \\
\vdots & & \vdots \\
\kappa_{\mu 1} & \ldots & \kappa_{\mu n}
\end{array}\right):=\boldsymbol{D C} \quad \text { and } \quad\left(p_{1}, \ldots, p_{\mu}\right):=\boldsymbol{h}+\boldsymbol{D} \boldsymbol{g}
$$

By $(16), \mathcal{B}_{\delta}:=\left\{\left(\kappa_{i 1}, \ldots, \kappa_{i n}, p_{i}\right)\right\}_{1 \leq i \leq \mu}$ spans a subspace of $\mathrm{V}\left(\boldsymbol{a}, \boldsymbol{f}_{\delta}, \mathbb{F}[t]_{\delta}\right)$. By linear algebra arguments it follows that $\mathcal{B}_{\delta}$ is a basis of $\mathrm{V}\left(\boldsymbol{a}, \boldsymbol{f}_{\delta}, \mathbb{F}[t]_{\delta}\right)$ over $\mathbb{K}$; see [39, Thm. 6.2].

Summarizing, with the above considerations we have proven Theorem 3.6 and hence Theorem 3.1.

Example 3.8 (Cont. Exp. 2.3) By Example 3.4 we have to find a basis $\mathcal{B}_{2}$ of $\mathrm{V}\left(\boldsymbol{a}, \boldsymbol{f}, \mathbb{F}[h]_{2}\right)$. Following our incremental reduction strategy, we look for a basis $\tilde{\mathcal{B}}_{2}$ of $\mathrm{V}\left(\tilde{\boldsymbol{a}}, \tilde{\boldsymbol{f}}_{2}, \mathbb{F}\right)$ with $\tilde{\boldsymbol{a}}_{2}:=\boldsymbol{a}$ and $\tilde{\boldsymbol{f}}_{2}=(0,0,0)$. We get $\tilde{\mathcal{B}}_{2}=\{(1,0,0,0),(0,1,0,0),(0,0,1,0),(0,0,0,1)\}$ which defines $\boldsymbol{C}_{2}=\mathbf{I d}_{3}$ and $\boldsymbol{g}_{2}=\left(0,0,0, h^{2}\right)$ This allows us to compute $\boldsymbol{f}_{1}:=\boldsymbol{C}_{2} \boldsymbol{f}-\sigma\left(\boldsymbol{g}_{2}\right)+\boldsymbol{g}_{2}=$ $\left(f_{1}^{\prime}, f_{2}^{\prime}, f_{2}^{\prime}, \frac{-1-2 h(1+k)}{(1+k)^{2}}\right)$ where $\boldsymbol{f}=\left(f_{1}^{\prime}, f_{2}^{\prime}, f_{3}^{\prime}\right)$. Now we have to compute a basis $\mathcal{B}_{1}$ of $\mathrm{V}\left(\boldsymbol{a}, \boldsymbol{f}_{1}, \mathbb{F}[t]_{1}\right)$. We start again our incremental reduction and compute a basis $\tilde{\mathcal{B}}_{1}$ of $\mathrm{V}\left(\tilde{\boldsymbol{a}}_{1}, \tilde{\boldsymbol{f}}_{1}, \mathbb{F}\right)$ with $\tilde{\boldsymbol{a}}_{1}:=\boldsymbol{a}$ and

$$
\tilde{f}_{1}=\left(b(-6 k+3 n), \frac{b(1+n)^{3}(3-6 k+3 n)}{(1-k+n)^{3}}, \frac{b(1+n)^{3}(2+n)^{3}(6-6 k+3 n)}{(1-k+n)^{3}(2-k+n)^{3}},-\left(\frac{2+2 k}{(1+k)^{2}}\right)\right) .
$$

In order to accomplish this task, we apply the same reduction technique for the extension $b$; see also Theorem 4.7. As result we obtain

$$
\begin{aligned}
\tilde{\mathcal{B}}_{1}= & \left\{\left\{-1,0,1,0, \frac{3 b k^{3}(3-k+2 n)\left(k^{3}-2(1+n)(2+n)-k^{2}(5+3 n)+k(9+n(11+3 n))\right)}{(1-k+n)^{3}(2-k+n)^{3}}\right\},\right. \\
& \left\{1, \quad 1,0,0, \frac{3 b k^{3}(2-k+2 n)}{(1-k+n)^{3}}\right\} \\
& \{0, \quad 0,0,0,1\}\} .
\end{aligned}
$$

This defines $\boldsymbol{C}_{1}$ by taking the first four columns and defines $\boldsymbol{g}_{1}$ by taking the last column multiplied with $h$. Next we compute $\boldsymbol{f}_{0}:=\boldsymbol{C}_{1} \boldsymbol{f}_{1}-\sigma\left(\boldsymbol{g}_{1}\right)+\boldsymbol{g}_{1}$ and 
get $\boldsymbol{f}_{0}=\left(f_{1}^{\prime \prime}, f_{2}^{\prime \prime}, f_{3}^{\prime \prime}\right)$ with

$$
\begin{gathered}
f_{1}^{\prime \prime}=\left(-b\left(4 k^{7}+6(1+n)^{3}(2+n)^{3}-2 k^{6}(19+15 n)+6 k^{5}(1+n)(25+16 n)\right.\right. \\
+3 k(1+n)^{2}(2+n)^{2}(-3+n(7+6 n))-3 k^{2}(1+n)(2+n)(31+n(109 \\
+n(103+29 n)))-k^{4}(315+n(807+n(651+167 n)))+k^{3}(363+n(1302 \\
\quad+n(1638+n(868+165 n))))) /\left((1+k)(1-k+n)^{3}(2-k+n)^{3}\right),
\end{gathered}
$$

$f_{2}^{\prime \prime}=-\frac{b\left(4 k^{4}+6 k^{2}(1+n)(3+5 n)+(1+n)^{3}(1+6 n)-2 k^{3}(7+9 n)-k(1+n)^{2}(11+23 n)\right)}{(1+k)(1-k+n)^{3}}$ and $f_{3}^{\prime \prime}=$ $-\frac{1}{1+k}$. Afterwards we have to look for a basis $\mathcal{B}_{0}$ of $\mathrm{V}\left(\boldsymbol{a}, \boldsymbol{f}_{0}, \mathbb{F}[h]_{0}\right)$. Following our reduction technique we look for a basis $\tilde{\mathcal{B}}_{0}$ of $\mathrm{V}\left(\tilde{\boldsymbol{a}}_{0}, \tilde{\boldsymbol{f}}_{0}, \mathbb{F}\right)$ where $\tilde{\boldsymbol{a}}_{0}:=\boldsymbol{a}$ and $\tilde{\boldsymbol{f}}_{0}:=\boldsymbol{f}_{0}$. We compute $\tilde{\mathcal{B}}_{0}=\{(n+2,2 n+3,0, w),(0,0,0,1)\}$ with

$$
\begin{gathered}
w=-b k^{2}(1+n)\left(2 k^{4}-6 k^{3}(3+2 n)+6(1+n)(2+n)^{2}(3+2 n)\right. \\
\left.+3 k^{2}(21+n(28+9 n))-2 k(2+n)(26+n(39+14 n))\right) /\left((1-k+n)^{3}(2-k+n)^{3}\right) .
\end{gathered}
$$

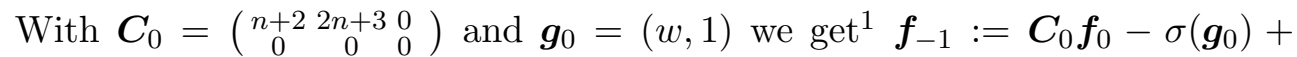
$\boldsymbol{g}_{0}=(0,0)$; a basis of $\mathrm{V}\left(\boldsymbol{a}, \boldsymbol{f}_{-1},\{0\}\right)$ is $\mathcal{B}_{-1}=\{(1,0,0),(0,1,0)\}$. This defines $\boldsymbol{D}_{-1}=\mathbf{I d}_{2}$ and $\boldsymbol{h}_{-1}=(0,0)$. To this end, we construct the basis $\mathcal{B}_{i}$ for $i=0,1,2$ by using (17). Namely, by $\boldsymbol{D}_{-1} \boldsymbol{C}_{0}=\boldsymbol{C}_{0}$ and $\boldsymbol{h}_{0}:=\boldsymbol{h}_{-1}+\boldsymbol{D}_{-1} \boldsymbol{g}_{0}=\boldsymbol{g}_{0}$

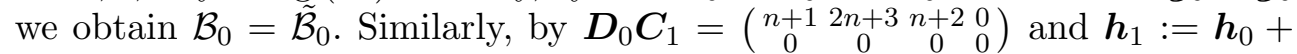
$\boldsymbol{D}_{0} \boldsymbol{g}_{1}=(g, 0)$ with (4) we get $\mathcal{B}_{1}=\{(n+1,2 n+3, n+2,0, g),(0,0,0,0,1)\}$. Finally, with $\boldsymbol{D}_{1} \boldsymbol{C}_{2}=\left(\begin{array}{ccc}n+1 & 2 n+3 & n+2 \\ 0 & 0 & 0\end{array}\right)$ and $\boldsymbol{h}_{2}=\boldsymbol{h}_{1}+\boldsymbol{D}_{1} \boldsymbol{g}_{2}=(g, 1)$ we arrive at $\mathcal{B}_{2}=\{(n+1,2 n+3, n+2, g),(0,0,0,1)\}$.

Example 3.9 (Cont. Exp. 2.4) By Example 3.5 we are interested in a nontrivial solution of $\mathrm{V}\left(\boldsymbol{a}, \boldsymbol{f}, \mathbb{F}[t]_{1}\right)$. First we look for a basis of $\mathrm{V}\left(\tilde{\boldsymbol{a}}_{1}, \tilde{\boldsymbol{f}}_{1}, \mathbb{F}\right)$ with $\tilde{\boldsymbol{a}}_{1}:=\boldsymbol{a}$ and

$$
\begin{gathered}
\tilde{\boldsymbol{f}}_{1}=(b(1+k)(2+k)(3+k)(k-n)(1-k+n), \\
-(b(1+k)(2+k)(3+k)(1+n)(1-k+n)),-(b(1+k)(2+k)(3+k)(1+n)(2+n))) .
\end{gathered}
$$

As a result we get $\tilde{\mathcal{B}}_{1}=\left\{\left(0,2,1,-\left(\frac{b\left(k+k^{2}\right)}{1-k+n}\right)\right),\left(2 n, n, 0, b\left(-k-k^{2}\right)\right)\right\}$, which provides us with two linearly independent solutions; see Example 5.5. According

${ }^{1}$ Here we could apply the shortcut in Sec. 3.3. In general, if $\boldsymbol{a} \notin \mathbb{F}^{m}$, we have to proceed as follows. 
to our reduction we obtain

$$
\begin{aligned}
\boldsymbol{f}_{0}=( & (1+k)(3+k)\left(2 k^{2}+3 n(1+n)-k(5+6 n)\right), \\
& \left.-\left(b(1+k)(3+k)(-1+k-n)\left(k+2 k^{2}-6 k n+3(-1+n) n\right)\right)\right) .
\end{aligned}
$$

Next, we look for a basis of $\mathrm{V}\left(\tilde{\boldsymbol{a}}_{0}, \tilde{\boldsymbol{f}}_{0}, \mathbb{F}\right)$ where $\tilde{\boldsymbol{a}}_{0}:=\boldsymbol{a}$ and $\tilde{\boldsymbol{f}}_{0}:=\boldsymbol{f}_{0}$. We get the solution $\tilde{\mathcal{B}}_{0}=\left\{\left(n^{2}(1+n)(2+n), 2 n(1+n)^{2}, w\right)\right\}$ with

$$
\begin{aligned}
w=b(1+k)\left(2 k^{2}(1+n)^{2}+n(1+n)\right. & (2+3 n(2+n)) \\
& -k(2+n(8+n(13+6 n)))) /(1-k+n) ;
\end{aligned}
$$

see Example 5.5. This defines ${ }^{1} \boldsymbol{f}_{-1}=(0)$. Next we take $\mathcal{B}_{-1}=\{(1,0)\}$ as basis of $\mathrm{V}\left(\boldsymbol{a}, \boldsymbol{f}_{-1},\{0\}\right)$. Finally, we get the linearly independent solutions $\mathcal{B}_{0}=\tilde{\mathcal{B}}_{0}$ of $\mathrm{V}\left(\boldsymbol{a}, \boldsymbol{f}_{0}, \mathbb{F}[h]_{0}\right)$ and $\mathcal{B}_{1}$ of $\mathrm{V}\left(\boldsymbol{a}, \boldsymbol{f}_{1}, \mathbb{F}[h]_{1}\right)$ as given in Example 3.5.

As indicated in the previous example, our reduction technique can be applied without having the property that the elements of $\tilde{\mathcal{B}}_{i}$ span the whole solution space $\mathrm{V}\left(\tilde{\boldsymbol{a}}_{i}, \tilde{\boldsymbol{f}}_{i}, \mathbb{F}\right)$. This observation will be considered further in Section 5 .

Definition 3.10 Let $(\mathbb{F}(t), \sigma)$ be a unimonomial extension of $(\mathbb{F}, \sigma), \mathbf{0} \neq \boldsymbol{a} \in$ $\mathbb{F}[t]^{m}, l:=\|\boldsymbol{a}\|$, and $\boldsymbol{f}=\boldsymbol{f}_{\delta} \in \mathbb{F}[t]_{\delta+l}^{n}$ for some $\delta \in \mathbb{N}_{0} \cup\{-1\}$. If we apply the reduction from above step by step, one obtains an incremental reduction of $\left(\boldsymbol{a}, \boldsymbol{f}, \mathbb{F}[t]_{\delta}\right)$ given in Figure 1 . We call $\left(\boldsymbol{f}_{\delta}, \ldots, \boldsymbol{f}_{-1}\right)$ the incremental problems and $\left(\left(\tilde{\boldsymbol{a}}_{\delta}, \tilde{\boldsymbol{f}}_{\delta}\right), \ldots,\left(\tilde{\boldsymbol{a}}_{0}, \tilde{\boldsymbol{f}}_{0}\right)\right)$ the coefficient problems.

In order to prove Theorem 5.7 we need the following results; the proof of the first lemma is immediate and is left to the reader.

LEMma 3.11 Let $(\mathbb{F}(t), \sigma)$ be a unimonomial extension of $(\mathbb{F}, \sigma)$ with $\mathbb{K}:=$ const $_{\sigma} \mathbb{F}, \mathbf{0} \neq \boldsymbol{a} \in \mathbb{F}[t]^{m}, \boldsymbol{f} \in \mathbb{F}[t]^{n}$ and $\boldsymbol{f}^{\prime}:=\boldsymbol{M} \boldsymbol{f} \in \mathbb{F}[t]^{n^{\prime}}$ for some $\boldsymbol{M} \in$ $\mathbb{K}^{n^{\prime} \times n}$. If $d \in \mathbb{F}[t]^{*}$ is a denominator bound of $\mathrm{V}(\boldsymbol{a}, \boldsymbol{f}, \mathbb{F}(t))$, $d$ is a denominator bound of $\mathrm{V}\left(\boldsymbol{a}, \boldsymbol{f}^{\prime}, \mathbb{F}(t)\right)$. If $b$ is a degree bound of $\mathrm{V}(\boldsymbol{a}, \boldsymbol{f}, \mathbb{F}[t]), b$ is a degree bound of $\mathrm{V}\left(\boldsymbol{a}, \boldsymbol{f}^{\prime}, \mathbb{F}[t]\right)$.

Lemma 3.12 Take $(\mathbb{F}, \sigma)$ with $\mathbb{K}:=$ const $_{\sigma} \mathbb{F}, \mathbf{0} \neq \boldsymbol{a} \in \mathbb{F}^{m}, \boldsymbol{f} \in \mathbb{F}^{n}$ and $\boldsymbol{f}^{\prime}:=\boldsymbol{M} \boldsymbol{f} \in \mathbb{F}^{n^{\prime}}$ with $\boldsymbol{M} \in \mathbb{K}^{n^{\prime} \times n}$. Let $\left\{\left(c_{i 1}, \ldots, c_{i n}, g_{i}\right)\right\}_{1 \leq i \leq \lambda}$ and $\left\{\left(c_{i 1}^{\prime}, \ldots, c_{i n^{\prime}}^{\prime}, g_{i}^{\prime}\right)\right\}_{1 \leq i \leq \lambda^{\prime}}\left(\lambda, \lambda^{\prime}>0\right)$ be bases of $\mathrm{V}(\boldsymbol{a}, \boldsymbol{f}, \mathbb{F})$ and $\mathrm{V}\left(\boldsymbol{a}, \boldsymbol{f}^{\prime}, \mathbb{F}\right)$, respectively. Define $\boldsymbol{C}=\left(c_{i j}\right) \in \mathbb{K}^{\lambda \times n}, \boldsymbol{g}=\left(g_{1}, \ldots, g_{\lambda}\right), \boldsymbol{C}^{\prime}=\left(c_{i j}^{\prime}\right) \in \mathbb{K}^{\lambda^{\prime} \times n^{\prime}}$, $\boldsymbol{g}^{\prime}=\left(g_{1}^{\prime}, \ldots, g_{\lambda^{\prime}}^{\prime}\right)$. Then there is $\boldsymbol{M}^{\prime} \in \mathbb{K}^{\lambda^{\prime} \times \lambda}$ with $\boldsymbol{C}^{\prime} \boldsymbol{M}=\boldsymbol{M}^{\prime} \boldsymbol{C}$ and $\boldsymbol{g}^{\prime}=\boldsymbol{M}^{\prime} \boldsymbol{g}$

Proof Suppose that $\boldsymbol{M}=\left(m_{i j}\right) \in \mathbb{K}^{n^{\prime} \times n}, \boldsymbol{f}=\left(f_{1}, \ldots, f_{n}\right), \boldsymbol{f}^{\prime}=\left(f_{1}^{\prime}, \ldots, f_{n^{\prime}}^{\prime}\right)$. Then $\sigma_{\boldsymbol{a}} g_{i}^{\prime}=\sum_{j=1}^{n^{\prime}} c_{i j}^{\prime} f_{j}^{\prime}=\sum_{j=1}^{n^{\prime}} c_{i j}^{\prime} \sum_{k=1}^{n} m_{j k} f_{k}=\sum_{k=1}^{n} f_{k} \sum_{j=1}^{n^{\prime}} c_{i j}^{\prime} m_{j k}$, and 
consequently we have $\left(\sum_{j=1}^{n^{\prime}} c_{i j}^{\prime} m_{j 1}, \ldots, \sum_{j=1}^{n^{\prime}} c_{i j}^{\prime} m_{j n}, g_{i}^{\prime}\right) \in \mathrm{V}(\boldsymbol{a}, \boldsymbol{f}, \mathbb{F})$. Since $\left\{\left(c_{i 1}, \ldots, c_{i n}, g_{i}\right)\right\}_{1 \leq i \leq \lambda}$ is a basis of $\mathrm{V}(\boldsymbol{a}, \boldsymbol{f}, \mathbb{F})$, we can take $\boldsymbol{M}^{\prime}=\left(m_{i j}^{\prime}\right) \in$ $\mathbb{K}^{\lambda^{\prime} \times \lambda}$ s.t. $g_{i}^{\prime}=\sum_{j=1}^{\lambda} m_{i j}^{\prime} g_{j}$ and $\sum_{j=1}^{n^{\prime}} c_{i j}^{\prime} m_{j k}=\sum_{j=1}^{\lambda} m_{i j}^{\prime} c_{j k}$ for all $i, k$ with $1 \leq i \leq \lambda^{\prime}$ and $1 \leq k \leq n$, i.e., $\boldsymbol{g}^{\prime}=\boldsymbol{M}^{\prime} \boldsymbol{g}$ and $\boldsymbol{C}^{\prime} \boldsymbol{M}=\boldsymbol{M}^{\prime} \boldsymbol{C}$.

Proposition 3.13 Let $(\mathbb{F}(t), \sigma)$ be a unimonomial extension of $(\mathbb{F}, \sigma), \mathbf{0} \neq$ $\boldsymbol{a} \in \mathbb{F}[t]^{m}, l:=\|\boldsymbol{a}\|, \boldsymbol{f} \in \mathbb{F}[t]_{\delta+l}^{n}$ for some $\delta \in \mathbb{N}_{0} \cup\{-1\}$ and $\boldsymbol{f}^{\prime}:=\boldsymbol{M} \boldsymbol{f}$ for some $\boldsymbol{M} \in \mathbb{K}^{n^{\prime} \times n}$. Let $\left(\boldsymbol{f}_{i}\right)_{-1 \leq i \leq \delta}$ (resp. $\left.\left(\boldsymbol{f}_{i}^{\prime}\right)_{-1 \leq i \leq \delta}\right)$ be the incremental problems and $\left\{\left(\tilde{\boldsymbol{a}}_{i}, \tilde{\boldsymbol{f}}_{i}\right)\right\}_{0 \leq i \leq \delta}$ (resp. $\left.\left\{\left(\tilde{\boldsymbol{a}}_{i}^{\prime}, \tilde{\boldsymbol{f}}_{i}^{\prime}\right)\right\}_{0 \leq i \leq \delta}\right)$ be the coefficient problems of an incremental reduction of $\left(\boldsymbol{a}, \boldsymbol{f}, \mathbb{F}[t]_{\delta}\right.$ ) (resp. of $\left(\boldsymbol{a}, \boldsymbol{f}^{\prime}, \mathbb{F}[t]_{\delta}\right)$ ). Then for $0 \leq i \leq \delta$ we have $\tilde{\boldsymbol{a}}_{i}=\tilde{\boldsymbol{a}}_{i}^{\prime}$ and there are $\boldsymbol{M}_{i} \in \mathbb{K}^{\lambda_{i}^{\prime} \times \lambda_{i}}$ such that $\boldsymbol{f}_{i}^{\prime}=\boldsymbol{M}_{i} \boldsymbol{f}_{i}$ and $\tilde{\boldsymbol{f}}_{i}^{\prime}=\boldsymbol{M}_{i} \tilde{\boldsymbol{f}}_{i}$. Moreover, $\boldsymbol{f}_{-1}^{\prime}=\boldsymbol{M}_{-1} \boldsymbol{f}_{-1}$ for some $\boldsymbol{M}_{-1} \in \mathbb{K}^{\lambda_{-1}^{\prime} \times \lambda_{-1}}$.

Proof By (12), $\tilde{\boldsymbol{a}}_{k}=\tilde{\boldsymbol{a}}_{k}^{\prime}$ is immediate for all $0 \leq i \leq \delta$. Moreover, by $\boldsymbol{f}_{\delta}=\boldsymbol{f}$ and $\boldsymbol{f}_{\delta}^{\prime}=\boldsymbol{f}^{\prime}$ we have $\boldsymbol{f}_{\delta}^{\prime}=\boldsymbol{M} \boldsymbol{f}_{\delta}$. If $\delta=-1$, we are done. Otherwise, suppose $\delta \geq 0$ and assume that we have proven the statement for all $r$ with $0 \leq$ $k \leq r \leq \delta$. Hence $\boldsymbol{f}_{k}^{\prime}=\boldsymbol{M}_{k} \boldsymbol{f}_{k}$ for some $\boldsymbol{M}_{k}=\left(m_{i j}\right) \in \mathbb{K}^{\lambda_{k}^{\prime} \times \lambda_{k}}$. Write $\boldsymbol{f}_{k}=$ $\left(h_{1}, \ldots, h_{\lambda_{k}}\right)$ and $\boldsymbol{f}_{k}^{\prime}=\left(h_{1}^{\prime}, \ldots, h_{\lambda_{k}^{\prime}}^{\prime}\right)$. Then by $\tilde{\boldsymbol{f}}_{k}=\left(\left[h_{1}\right]_{k+l}, \ldots,\left[h_{\lambda_{k}}\right]_{k+l}\right)$ and $\left[h_{i}^{\prime}\right]_{k+l}=\left[\sum_{j=1}^{\lambda_{k}} m_{i j} h_{j}\right]_{k+l}=\sum_{j=1}^{\lambda_{k}} m_{i j}\left[h_{j}\right]_{k+l}$ for $1 \leq i \leq \lambda_{k}, \tilde{\boldsymbol{f}}_{k}^{\prime}=\boldsymbol{M}_{k} \tilde{\boldsymbol{f}}_{k}$.

Within the two incremental reductions suppose that we have obtained the bases $\left\{\left(c_{i 1}, \ldots, c_{i \lambda_{k}}, w_{i}\right)\right\}_{1 \leq i \leq \lambda_{k-1}}\left(\lambda_{k-1} \geq 0\right)$ and $\left\{\left(c_{i 1}^{\prime}, \ldots, c_{i \lambda_{k}^{\prime}}^{\prime}, w_{i}^{\prime}\right)\right\}_{1 \leq i \leq \lambda_{k-1}^{\prime}}$ $\left(\lambda_{k-1}^{\prime} \geq 0\right)$ of $\mathrm{V}\left(\tilde{\boldsymbol{a}}_{k}, \tilde{\boldsymbol{f}}_{k}, \mathbb{F}\right)$ and $\mathrm{V}\left(\tilde{\boldsymbol{a}}_{k}, \tilde{\boldsymbol{f}}_{k}^{\prime}, \mathbb{F}\right)$, respectively.

First suppose that $\lambda_{k-1}=\lambda_{k-1}^{\prime}=0$, i.e., we are in case I in both situations. Then $\boldsymbol{f}_{k-1}=\boldsymbol{f}_{k-1}^{\prime}=(0)$, i.e., we can choose $\boldsymbol{M}_{k-1}=(1)$ in order to get $\boldsymbol{M}_{k-1} \boldsymbol{f}_{k-1}=\boldsymbol{f}_{k-1}^{\prime}$. Now suppose that $\lambda_{k-1}=0$ (case I), but $\lambda_{k-1}^{\prime}>0$ (case II). Define $\boldsymbol{C}^{\prime}=\left(c_{i j}^{\prime}\right) \in \mathbb{K}^{\lambda_{k-1}^{\prime} \times \lambda_{k}^{\prime}}$. It follows that $\left(c_{i 1}^{\prime}, \ldots, c_{i \lambda_{k^{\prime}}^{\prime}}^{\prime}\right) \boldsymbol{M}_{k}=\mathbf{0}$. Hence, we get $\tilde{\boldsymbol{f}}_{k-1}^{\prime}=\boldsymbol{C}^{\prime} \boldsymbol{f}_{k}^{\prime}=\boldsymbol{C}^{\prime} \boldsymbol{M}_{k} \boldsymbol{f}_{k}=\mathbf{0}$ by following (15). Thus we can choose $M_{k-1}=(0, \ldots, 0) \in \mathbb{K}^{1 \times \lambda_{k-1}}$ in order to get $\boldsymbol{f}_{k-1}^{\prime}=\boldsymbol{M}_{k-1} \boldsymbol{f}_{k-1}^{\prime}$. If $\lambda_{k-1}>0$ (case II) and $\lambda_{k-1}^{\prime}=0$ (case I) we have $\boldsymbol{f}_{k-1}^{\prime}=(0)$, and we can choose $\boldsymbol{M}_{k-1}=(0, \ldots, 0) \in \mathbb{K}^{1 \times \lambda_{k-1}}$ s.t. $\boldsymbol{f}_{k-1}^{\prime}=\boldsymbol{M}_{k-1} \boldsymbol{f}_{k-1}$. Otherwise, suppose that $\lambda_{k-1}, \lambda_{k-1}^{\prime}>0$, i.e., we are in case II in both situations. Define $\boldsymbol{C}=$ $\left(c_{i j}\right) \in \mathbb{K}^{\lambda_{k-1} \times \lambda_{k}}, \boldsymbol{C}^{\prime}=\left(c_{i j}^{\prime}\right) \in \mathbb{K}^{\lambda_{k-1}^{\prime} \times \lambda_{k}^{\prime}}, \boldsymbol{g}=\left(w_{1} t^{k}, \ldots, w_{\lambda_{k-1}} t^{k}\right)$ and $\boldsymbol{g}^{\prime}=$ $\left(w_{1}^{\prime} t^{k}, \ldots, w_{\lambda_{k-1}^{\prime}}^{\prime} t^{k}\right)$. Then by Lemma 3.12 there is an $\boldsymbol{M}_{k-1} \in \mathbb{K}^{\lambda_{k-1}^{\prime} \times \lambda_{k-1}}$ with $\boldsymbol{C}^{\prime} \boldsymbol{M}_{k}=\boldsymbol{M}_{k-1} \boldsymbol{C}$ and $\boldsymbol{g}^{\prime}=\boldsymbol{M}_{k-1} \boldsymbol{g}$. Hence

$$
\begin{aligned}
\boldsymbol{f}_{k-1}^{\prime}=\boldsymbol{C}^{\prime} \boldsymbol{f}_{k}^{\prime}-\sigma_{\boldsymbol{a}} \boldsymbol{g}^{\prime}= & \boldsymbol{C}^{\prime} \boldsymbol{M}_{k} \boldsymbol{f}_{k}-\sigma_{\boldsymbol{a}} \boldsymbol{g}^{\prime} \\
& =\boldsymbol{M}_{k-1} \boldsymbol{C} \boldsymbol{f}_{k}-\sigma_{\boldsymbol{a}}\left(\boldsymbol{M}_{k-1} \boldsymbol{g}\right)=\boldsymbol{M}_{k-1}\left(\boldsymbol{C} \boldsymbol{f}_{k}-\sigma_{\boldsymbol{a}} \boldsymbol{g}\right)
\end{aligned}
$$


and therefore $\boldsymbol{f}_{k-1}^{\prime}=\boldsymbol{M}_{k-1} \boldsymbol{f}_{k-1}$. This finishes the induction step.

Proposition 3.13 implies that there are invertible $\boldsymbol{M}_{i}$ if $\boldsymbol{M}$ is invertible. In particular, by choosing $\boldsymbol{M}=\mathbf{I d}_{n}$ it follows that the incremental and coefficients problems of a reduction of $\left(\boldsymbol{a}, \boldsymbol{f}, \mathbb{F}[t]_{\delta}\right)$ are uniquely determined up to the multiplication with invertible matrices $\boldsymbol{M}_{i}$.

\subsection{Some remarks}

The following approaches can be related to our reduction technique.

- In Karr's approach [18] reduction techniques have been developed that solve problem $P L D E$ with $\mathbf{0} \neq \boldsymbol{a} \in \mathbb{F}(t)^{2}$. More precisely, the solutions $g=$ $p+q \in \mathbb{F}[t] \oplus \mathbb{F}(t)^{(\text {frac })}$ in $\left(c_{1}, \ldots, c_{n}, g\right) \in \mathrm{V}(\boldsymbol{a}, \boldsymbol{f}, \mathbb{F}(t))$ are computed by deriving first the polynomial part $p$ and afterwards finding the fractional part $q$. We have simplified this approach by first looking for a common denominator of all the possible solutions in $\mathbb{F}(t)$ and afterwards computing the "numerator" of the solutions over this common denominator. Moreover, we have generalized Karr's reduction techniques to the case $\mathbf{0} \neq \boldsymbol{a} \in \mathbb{F}(t)^{m}$.

- As a side remark note that similar reduction techniques have been used in [24, Lemma 3.2] in order to solve linear differential equations with Liouvillian coefficients.

- In [23, Thm. 1] reduction techniques have been developed for problem PLDE in monomial extensions. Monomial extensions cover besides unimonomial difference and differential field extensions for instance difference algebras of the type $(\mathbb{F}(t), \sigma)$ where $\mathbb{F}(t)$ is a rational function field and $\sigma: \mathbb{F} \rightarrow \mathbb{F}$ is an epimorphism with $\sigma(t) \in \mathbb{F}[t]^{*}$. But there is one restriction in this approach: one needs a polynomial $p \in \mathbb{F}[t] \backslash \mathbb{F}$ with $\frac{\sigma(p)}{p} \in \mathbb{F}[t]$ in which the solutions are expanded. By [18, Thm. 4] such an element $p$ exists if $t$ is a П-extension, but does not exist if $t$ is a $\Sigma$-extension. Hence our approach, which can handle also $\Sigma$-extensions (Theorem 3.6), is an essential contribution in the context of multi-summation.

Restricting to $\Pi$-extensions, the reduction strategy in [23, Thm. 1] can be simplified to our strategy, besides the fact that in our approach we compute the leading coefficient first and then the coefficients of lower degree, and in the approach [23] one starts looking for the constant coefficient and then derives the remaining coefficients of higher degree; note that one could even compute the coefficients simultaneously without imposing any order. 


\section{A recursive algorithm for unimonomial and $\Pi \Sigma$-extensions}

Applying Theorem 3.1 recursively we arrive at Theorem 4.2 for the following type of unimonomial extensions.

Definition 4.1 A unimonomial extension $\left(\mathbb{G}\left(t_{1}\right) \ldots\left(t_{e}\right), \sigma\right)$ of $(\mathbb{G}, \sigma)$ with $\mathbb{K}:=$ const $_{\sigma} \mathbb{G}$ is called $r$-solvable $(r \geq 0)$ if one can solve problem PLDE in $(\mathbb{G}, \sigma)$ and for all $i$ and $m$ with $1 \leq i \leq e$ and $2 \leq m \leq r+1$ the following holds. One can solve problems $\operatorname{Den} B$ and $\operatorname{Deg} B$ in the unimonomial extension $t_{i}$, and one can solve problem $N S$ in $\mathbb{G}\left(t_{1}\right) \ldots\left(t_{i}\right)$.

ThEOREM 4.2 Let $\left(\mathbb{G}\left(t_{1}\right) \ldots\left(t_{e}\right), \sigma\right)$ be a unimonomial extension of $(\mathbb{G}, \sigma)$ which is $r$-solvable. Then there is an algorithm that solves parameterized linear difference equations of order $r$, i.e., solves problem PLDE with $m=r+1$.

More precisely, the resulting algorithm can be stated as follows.

Algorithm 4.3 SolveSolutionSpace $\left(\boldsymbol{a}, \boldsymbol{f},\left(\mathbb{G}\left(t_{1}\right) \ldots\left(t_{e}\right), \sigma\right)\right)$

Input: An $(m-1)$-solvable unimonomial extension $\left(\mathbb{G}\left(t_{1}\right) \ldots\left(t_{e}\right), \sigma\right)$ of $(\mathbb{G}, \sigma)$ with $\mathbb{K}:=$ const $_{\sigma} \mathbb{G} ; \mathbf{0} \neq \boldsymbol{a}=\left(a_{1}, \ldots, a_{m}\right) \in \mathbb{G}\left(t_{1}\right) \ldots\left(t_{e}\right)^{m}$ and $\boldsymbol{f} \in \mathbb{G}\left(t_{1}\right) \ldots\left(t_{e}\right)^{n}$.

Output: A basis of $\mathrm{V}\left(\boldsymbol{a}, \boldsymbol{f}, \mathbb{G}\left(t_{1}\right) \ldots\left(t_{e}\right)\right)$.

(1) IF $e=0$, compute a basis $\mathcal{B}$ of $\mathrm{V}(\boldsymbol{a}, \boldsymbol{f}, \mathbb{G})$; RETURN $\mathcal{B}$. FI

Let $\mathbb{F}:=\mathbb{G}\left(t_{1}\right) \ldots\left(t_{e-1}\right)$, i.e., $\left(\mathbb{F}\left(t_{e}\right), \sigma\right)$ is a unimonomial extension of $(\mathbb{F}, \sigma)$.

(*A Simplification and shortcut: Subsection $3.1^{*}$ )

(2) Define $l, k$ as in Simplification I. Transform $\boldsymbol{a}, \boldsymbol{f}$ by (7) to $\boldsymbol{a}^{\prime}=\left(a_{1}^{\prime}, \ldots, a_{m^{\prime}}^{\prime}\right) \in$ $\mathbb{F}\left(t_{e}\right)^{m^{\prime}}, \boldsymbol{f}^{\prime} \in \mathbb{F}\left(t_{e}\right)^{n}$ with $a_{1}^{\prime} a_{m^{\prime}}^{\prime} \neq 0, m^{\prime} \leq m$; clear denominators and common factors s.t. $\boldsymbol{a}^{\prime} \in \mathbb{F}\left[t_{e}\right]^{m^{\prime}}, \boldsymbol{f}^{\prime} \in \mathbb{F}\left[t_{e}\right]^{n}$. FI

(3) IF $\boldsymbol{a}^{\prime} \in \mathbb{F}\left[t_{e}\right]^{1}$, set $\left(g_{1}, \ldots, g_{n}\right):=\frac{f}{a_{1}^{\prime}} ; \operatorname{RETURN}\left\{\left(0 \ldots, 1, \ldots, 0, \sigma^{m-k}\left(g_{i}\right)\right)\right\}_{1 \leq i \leq n}$ where $(0, \ldots, 1, \ldots, 0)$ is the $i$ th unit vector. FI

(*Bounds for the solution space: Subsection $3.2^{*}$ )

(4) Compute a denominator bound $d \in \mathbb{F}\left[t_{e}\right]^{*}$ of $\mathrm{V}\left(\boldsymbol{a}^{\prime}, \boldsymbol{f}^{\prime}, \mathbb{F}\left(t_{e}\right)\right)$.

(5) Set $\boldsymbol{a}^{\prime \prime}:=\left(\frac{a_{1}^{\prime}}{\sigma^{m^{\prime}-1}(d)}, \ldots, \frac{a_{m^{\prime}}}{d}\right) \in \mathbb{F}\left(t_{e}\right)^{m^{\prime}}, \boldsymbol{f}^{\prime \prime}:=\boldsymbol{f}^{\prime}$, and clear denominators and common factors s.t. $\boldsymbol{a}^{\prime \prime} \in \mathbb{F}\left[t_{e}\right]^{m^{\prime}}$ and $\boldsymbol{f}^{\prime \prime} \in \mathbb{F}\left[t_{e}\right]^{n}$.

(6) Compute a degree bound $b$ of $\mathrm{V}\left(\boldsymbol{a}^{\prime \prime}, \boldsymbol{f}^{\prime \prime}, \mathbb{F}\left[t_{e}\right]\right)$.

(*Incremental reduction: Subsection $3.3^{*}$ )

(7) Compute $\mathcal{B}:=\operatorname{IncrementalReduction}\left(\boldsymbol{a}^{\prime}, \boldsymbol{f}^{\prime},\left(\mathbb{F}\left(t_{e}\right), \sigma\right), b\right)$; suppose we obtain $\mathcal{B}=$ $\left\{\left(\kappa_{i 1}, \ldots, \kappa_{i n}, p_{i}\right)\right\}_{1 \leq i \leq \mu}$.

(8) IF $\mathcal{B}=\{\}$ THEN RETURN \{\} ELSE RETURN $\left\{\left(\kappa_{i 1}, \ldots, \kappa_{i n}, \sigma^{m-k}\left(\frac{p_{i}}{d}\right)\right)\right\}_{1 \leq i \leq \mu}$. FI

Algorithm 4.4 IncrementalReduction $\left(\left(\boldsymbol{a}, \boldsymbol{f}, \mathbb{G}\left(t_{1}\right) \ldots\left(t_{e}\right)(t), \sigma\right), \delta\right)$

Input: An $(m-1)$-solvable unimonomial extension $(\mathbb{F}(t), \sigma)$ of $(\mathbb{G}, \sigma)$ with $\mathbb{K}:=$ const $_{\sigma} \mathbb{G}$ and $\mathbb{F}:=\mathbb{G}\left(t_{1}\right) \ldots\left(t_{e}\right) ; \delta \in \mathbb{N}_{0} \cup\{-1\} ; \mathbf{0} \neq \boldsymbol{a} \in \mathbb{F}[t]^{m}$ with $l:=\|\boldsymbol{a}\|$, and $\boldsymbol{f} \in \mathbb{F}[t]_{l+\delta}^{n}$.

Output: A basis of $\mathrm{V}\left(\boldsymbol{a}, \boldsymbol{f}, \mathbb{F}[t]_{\delta}\right)$ over $\mathbb{K}$. 
(1) IF $\delta=-1$, RETURN a basis of Nullspace $_{\mathbb{K}}(\boldsymbol{f}) \times\{0\}$ over $\mathbb{K}$. FI

(2) Define $\mathbf{0} \neq \tilde{\boldsymbol{a}}_{\delta} \in \mathbb{F}^{m}$ and $\tilde{\boldsymbol{f}}_{\delta} \in \mathbb{F}^{n}$ as in (12).

(3) Compute $\tilde{\mathcal{B}}:=$ SolveSolutionSpace $\left(\tilde{\boldsymbol{a}}_{\delta}, \tilde{\boldsymbol{f}}_{\delta},(\mathbb{F}, \sigma)\right)$.

(4) IF $\tilde{\mathcal{B}}=\{\}$ THEN

(5) Compute $\mathcal{B}:=$ IncrementalReduction $(\boldsymbol{a},(0),(\mathbb{F}(t), \sigma), \delta-1)$.

Extract a basis, say $H=\left\{g_{1}, \ldots, g_{\mu}\right\}$, for $(13)$ from $\mathcal{B}$.

(6) IF $H=\{\}$ THEN RETURN \{\} ELSE RETURN $\left\{\left(0, \ldots, 0, g_{i}\right)\right\}_{1 \leq i \leq \mu}$. FI FI

(7) Given $\tilde{\mathcal{B}}=\left\{\left(c_{i 1}, \ldots, c_{i n}, w_{i}\right)\right\}_{1 \leq i \leq \lambda}$, take $\boldsymbol{C}=\left(c_{i j}\right) \in \mathbb{K}^{\lambda \times n}, \boldsymbol{g} \in t^{\delta} \mathbb{F}^{\lambda}, \boldsymbol{f}_{\delta-1} \in$ $\mathbb{F}[t]_{\delta-1}^{\lambda}$ as in (14), (15).

(8) Compute $\mathcal{B}:=\operatorname{IncrementalReduction}\left(\boldsymbol{a}, \boldsymbol{f}_{\delta-1},(\mathbb{F}(t), \sigma), \delta-1\right)$.

(9) IF $\mathcal{B}=\{\}$ THEN RETURN \{\} FI

(10) Given $\mathcal{B}=\left\{\left(d_{i 1}, \ldots, d_{i \lambda}, h_{i}\right)\right\}_{1<i<\mu}$, take $\boldsymbol{D}:=\left(d_{i j}\right) \in \mathbb{K}^{\mu \times \lambda}, \boldsymbol{h}:=\left(h_{1}, \ldots, h_{\mu}\right) \in$ $\mathbb{F}[t]_{\delta-1}^{\mu}$. Take $\kappa_{i j} \in \mathbb{K}$ for $1 \leq i \leq \mu, 1 \leq j \leq n$, and take $p_{i} \in \mathbb{F}[t]_{\delta}$ for $1 \leq i \leq \mu$ as in (17). RETURN $\left\{\left(\kappa_{i 1}, \ldots, \kappa_{i n}, p_{i}\right)\right\}_{1 \leq i \leq \mu}$

By Lemma 3.2 and Theorem 4.2 we get the following result.

Corollary 4.5 Let $\left(\mathbb{K}\left(t_{1}\right) \ldots\left(t_{e}\right), \sigma\right)$ be a unimonomial field over a $\sigma$ computable $\mathbb{K}$, and let $r \geq 0$. If for all $m, i$ with $2 \leq m \leq r+1$ and $1 \leq i \leq e$ one can solve problem DegB and DenB in the unimonomial extension $t_{i}$, then $\left(\mathbb{K}\left(t_{1}\right) \ldots\left(t_{e}\right), \sigma\right)$ is $r$-solvable, i.e., one can solve parameterized linear difference equations of order $r$.

In [1-7] various algorithms are developed that solve problems $\operatorname{Deg} B$ and $\operatorname{Den} B$ for the rational case and its $q-$ analog version. All these results immediately lead to the following

TheOREM 4.6 Let $\mathbb{K}$ and $\mathbb{K}(q)$ ( $q$ transcendental) be $\sigma$-computable fields. Then the $\Pi \Sigma$-field $(\mathbb{K}(k), \sigma)$ with $\sigma(k)=k+1$ and the $\Pi \Sigma$-field $(\mathbb{K}(q)(x), \sigma)$ with $\sigma(x)=q x$ are $r$-solvable.

Suppose that $(\mathbb{F}(t), \sigma)$ is a $\Pi \Sigma$-field over a $\sigma$-computable $\mathbb{K}$. Then by $[18,23]$ one can solve problem $\operatorname{Den} B$ if $t$ is a $\Sigma$-extension, and by [18] one can solve problem $\operatorname{Deg} B$ with $m=2$ if $t$ is a $\Pi \Sigma$-extension; for proofs and algorithms see $[25,26]$. This shows

THEOREM 4.7 Any $\Pi \Sigma$-field $(\mathbb{F}, \sigma)$ over a $\sigma$-computable constant field $\mathbb{K}$ is 1-solvable, i.e., one can solve first order parameterized linear difference equations.

Remark 4.8 The following remarks are in place.

- The resulting algorithm from Theorem 4.7 is a simplified version of [18]. These simplifications were the starting point to derive refined and extended summation algorithms in [27-29]. All these algorithms are implemented in our package Sigma. 
- Various special cases of $\operatorname{DenB}$ and $\operatorname{DegB}$ have been solved in [25,26]. Furthermore, methods have been developed in [10] that find degree bounds for $\Sigma^{*}$-extensions. Hence only $\Pi$-extensions and $\Sigma$-extensions that are not $\Sigma^{*}$ extensions remain as problematic cases. A challenging task is to solve problem $\operatorname{Den} B$ and $\operatorname{DegB}$ in full generality. This would turn Algorithm 4.3 to a complete algorithm for $\Pi \Sigma$-fields.

\section{$5 \quad$ Finding all solutions of problem $P L D E$ in $\Pi \mathbf{\Pi}$-fields}

The algorithm presented in the previous section cannot be applied for general $\Pi \Sigma$-fields since the two subproblems $\operatorname{Den} B$ and $\operatorname{Deg} B$ have not been solved in full generality so far. To overcome this problem, we shall modify our algorithm to a version that can be executed if one can solve a weakened version of problem $\operatorname{Den} B$, namely $W D e n B$. With this algorithm one usually cannot solve problem PLDE, but one can look at least for solutions of problem PLDE, see Exp. 3.9. Applying this algorithm iteratively, one eventually finds all solutions of problem $P L D E$.

More precisely, we adapt Algorithms 4.3 and 4.4 as follows. Suppose that we are given a $\Pi \Sigma$-extension $\left(\mathbb{G}\left(t_{1}\right) \ldots\left(t_{e}\right), \sigma\right)$ of $(\mathbb{G}, \sigma)$ where problem $W D e n B$ is solvable for each extension $t_{i}$.

WDenB: Weak Denominator Bounding.

- Given a $\Pi \Sigma$-extension $(\mathbb{F}(t), \sigma)$ of $(\mathbb{F}, \sigma)$ with $\mathbb{K}:=$ const $_{\sigma} \mathbb{F} ; \mathbf{0} \neq \boldsymbol{a} \in \mathbb{F}[t]^{m}$ and $f \in \mathbb{F}[t]^{n}$.

- Find a weak denominator bound of $\mathrm{V}(\boldsymbol{a}, \boldsymbol{f}, \mathbb{F}(t))$, i.e., a polynomial $d \in \mathbb{F}[t]^{*}$ with the following properties. If $t$ is a $\Sigma$-extension, $d$ is a denominator bound of $\mathrm{V}(\boldsymbol{a}, \boldsymbol{f}, \mathbb{F}(t))$. Otherwise there is an $x \in \mathbb{N}_{0}$ such that $t^{x} d$ is a denominator bound of $\mathrm{V}(\boldsymbol{a}, \boldsymbol{f}, \mathbb{F}(t))$.

Remark. This is possible if $(\mathbb{G}, \sigma)$ itself is a $\Pi \Sigma$-field over a $\sigma$-computable $\mathbb{K}$; see Theorem 5.2.

Then one can guess an $x \in \mathbb{N}_{0}$ to complete the denominator bound and can guess a degree bound $y \in \mathbb{N}_{0}$ in order to simulate Algorithm 4.3.

Namely, let $(\mathbb{F}(t), \sigma)$ be a $\Pi \Sigma$-extension of $(\mathbb{F}, \sigma)$ with $\mathbf{0} \neq \boldsymbol{a}^{\prime} \in \mathbb{F}[t]^{m^{\prime}}$ and $f^{\prime} \in \mathbb{F}[t]^{n}$.

Approximation of a denominator. Suppose that we have computed a weak denominator bound $d^{\prime} \in \mathbb{F}[t]^{*}$ of $\mathrm{V}\left(\boldsymbol{a}^{\prime}, \boldsymbol{f}^{\prime}, \mathbb{F}(t)\right)$. Then we try to take an $x \in \mathbb{N}_{0}$, as in line (4) of Algorithm 5.3, such that $d:=d^{\prime} t^{x}$ is a denominator bound of $\mathrm{V}\left(\boldsymbol{a}^{\prime}, \boldsymbol{f}^{\prime}, \mathbb{F}(t)\right)$.

Approximation of a degree bound. After computing $\boldsymbol{a}^{\prime \prime}$ and $\boldsymbol{f}^{\prime \prime}$ as in line (5), one is faced with the problem to choose a $b$ that approximates a degree bound of $\mathrm{V}\left(\boldsymbol{a}^{\prime \prime}, \boldsymbol{f}^{\prime \prime}, \mathbb{F}[t]\right)$.

For instance, fix $y \geq 0$, and take a $b$ with the following property: If one can compute all solutions of $\mathrm{V}\left(\boldsymbol{a}^{\prime \prime}, \boldsymbol{f}^{\prime \prime}, \mathbb{F}[t]_{b}\right)$, one should be able to reconstruct all 
solutions of $\mathrm{V}\left(\boldsymbol{a}^{\prime}, \boldsymbol{f}^{\prime}, \mathbb{F}[t]_{y} \oplus \mathbb{F}(t)^{(\text {frac })}\right)$ by executing line (8).

Remark. Suppose that we have managed to obtain a denominator bound $d$ of $\mathrm{V}(\boldsymbol{a}, \boldsymbol{f}, \mathbb{F}(t))$ by the strategy explained above. Then $\left\{\left(c_{i 1}, \ldots, c_{i n}, g_{i}\right)\right\}_{1 \leq i \leq r}$ is a basis of $\mathrm{V}\left(\boldsymbol{a}^{\prime \prime}, \boldsymbol{f}^{\prime \prime}, \mathbb{F}[t]_{b}\right)$ if and only if $\left\{\left(c_{i 1}, \ldots, c_{i n}, \frac{g_{i}}{d}\right)\right\}_{1 \leq i \leq r}$ is a basis of $\mathrm{V}\left(\boldsymbol{a}^{\prime}, \boldsymbol{f}^{\prime}, \mathbb{F}[t]_{b-\operatorname{deg}(d)} \oplus \mathbb{F}(t)^{(\text {frac })}\right)$; see [39, Thm. 7.6].

This result motivates us to take the approximated degree bound $b:=y+$ $\|d\|$ of $\mathrm{V}\left(\boldsymbol{a}^{\prime \prime}, \boldsymbol{f}^{\prime \prime}, \mathbb{F}[t]\right)$, i.e., we try to look for a basis of the solution space $\mathrm{V}\left(\boldsymbol{a}^{\prime}, \boldsymbol{f}^{\prime}, \mathbb{F}[t]_{y} \oplus \mathbb{F}(t)^{(\text {frac })}\right)$.

Another strategy is to look at the number $y$ of the highest possible coefficients that cancel in $\sigma_{\boldsymbol{a}^{\prime \prime}} g=: f \in \mathbb{F}[t]$, i.e., $\left\|\boldsymbol{a}^{\prime \prime}\right\|+\|g\|=\|f\|+y$. Following this idea, we should fix $y$ and take $b:=y+\max \left(\left\|\boldsymbol{f}^{\prime \prime}\right\|-\left\|\boldsymbol{a}^{\prime \prime}\right\|, 0\right)$ as the degree bound. Combining both aspects gives the approximated degree bound

$$
b:=y+\max \left(\left\|\boldsymbol{f}^{\prime \prime}\right\|-\left\|\boldsymbol{a}^{\prime \prime}\right\|,\|d\|\right)
$$

for a fixed $y \geq 0$; see line (6) of Algorithm 5.3. Note that in the implementation of Sigma we used the bound (18).

In order to apply our approximated reduction recursively, the definition of weak $r$-solvable $\Pi \Sigma$-extensions is introduced in which one can solve problem $W D e n B$ for each extension $t_{i}$. Moreover we define a bounding matrix that specifies these tuples $(x, y)$ for each extension $t_{i}$.

Definition 5.1 Let $\left(\mathbb{F}\left(t_{1}\right) \ldots\left(t_{e}\right), \sigma\right)$ be a $\Pi \Sigma$-extension of $(\mathbb{F}, \sigma)$ with $\mathbb{K}:=$ const $_{\sigma} \mathbb{F}$. This extension is called weak $r$-solvable $(r \geq 0)$ if one can solve problem $P L D E$ in $(\mathbb{F}, \sigma)$ and for all $i$ and $m$ with $1 \leq i \leq e$ and $2 \leq m \leq r+1$ the following holds: One can solve problems $W \operatorname{Den} B$ in the $\Pi \Sigma$-extension $t_{i}$, and one can solve problem $N S$ in $\mathbb{F}\left(t_{1}\right) \ldots\left(t_{i}\right)$.

We call $\left(\begin{array}{ccc}x_{1} & \ldots & x_{e} \\ y_{1} & \ldots & y_{e}\end{array}\right) \in \mathbb{N}_{0}^{2 \times e}$ a bounding matrix of length e for $\mathbb{F}\left(t_{1}\right) \ldots\left(t_{e}\right)$, if for all $1 \leq i \leq e$ we have $x_{i}=0$ when $t_{i}$ is a $\Sigma$-extension. (If $e=0$, the bounding matrix is defined as ().)

Let $(\mathbb{F}(t), \sigma)$ be a $\Pi \Sigma$-field over a $\sigma$-computable $\mathbb{K}$. Then by $[25$, Theorem 6.4], see also [23], there is an algorithm that solves problem WDenB. With Lemma 3.2 we get

THEOREM 5.2 A $\Pi \Sigma$-field over a $\sigma$-computable constant field is weak $r$ solvable.

Summarizing, we obtain the following algorithms that can be applied in $\Pi \Sigma$ fields.

Algorithm 5.3 SolveSolutionSpaceH $\left(\boldsymbol{a}, \boldsymbol{f},\left(\mathbb{G}\left(t_{1}\right) \ldots\left(t_{e}\right), \sigma\right), \boldsymbol{B}\right)$

Input: A weak $(m-1)$-solvable $\Pi \Sigma$-extension $\left(\mathbb{G}\left(t_{1}\right) \ldots\left(t_{e}\right), \sigma\right)$ of $(\mathbb{G}, \sigma)$ with $\mathbb{K}:=$ 
const $_{\sigma} \mathbb{G} ;$ a bounding matrix $\boldsymbol{B}$ of length $e$ for $\mathbb{G}\left(t_{1}\right) \ldots\left(t_{e}\right), \mathbf{0} \neq \boldsymbol{a}=\left(a_{1}, \ldots, a_{m}\right) \in$ $\mathbb{G}\left(t_{1}\right) \ldots\left(t_{e}\right)^{m}$ and $\boldsymbol{f} \in \mathbb{G}\left(t_{1}\right) \ldots\left(t_{e}\right)^{n}$.

Output: A basis of a subspace of $\mathrm{V}\left(\boldsymbol{a}, \boldsymbol{f}, \mathbb{G}\left(t_{1}\right) \ldots\left(t_{e}\right)\right)$ over $\mathbb{K}$.

Exactly the same lines as in Algorithm 4.3, but replacing lines (4), (6) and (7) with:

(4) Let $\boldsymbol{B}=\left(\begin{array}{llll}x_{1} & \ldots & x_{e-1} & x \\ y_{1} & \ldots & y_{e-1} & y\end{array}\right)$ and set $\boldsymbol{B}_{0}:=\left(\begin{array}{lll}x_{1} & \ldots & x_{e-1} \\ y_{1} & \ldots & y_{e-1}\end{array}\right)$; if $e=1, \boldsymbol{B}_{0}$ is the empty list. Compute a weak denominator bound $d^{\prime} \in \mathbb{F}\left[t_{e}\right]^{*}$ of $\mathrm{V}\left(\boldsymbol{a}^{\prime}, \boldsymbol{f}^{\prime}, \mathbb{F}(t)\right)$ and approximate a denominator bound by setting $d:=d^{\prime} t_{e}^{x}$.

(6) Approximate a degree bound by setting $b:=y+\max \left(\left\|\boldsymbol{f}^{\prime \prime}\right\|-\left\|\boldsymbol{a}^{\prime \prime}\right\|,\|d\|\right)$.

(7) Compute $\mathcal{B}:=\operatorname{IncrementalReductionH}\left(\boldsymbol{a}^{\prime}, \boldsymbol{f}^{\prime},\left(\mathbb{F}\left(t_{e}\right), \sigma\right), b, \boldsymbol{B}_{0}\right)$; suppose we obtained $\mathcal{B}=\left\{\left(\kappa_{i 1}, \ldots, \kappa_{i n}, p_{i}\right)\right\}_{1 \leq i \leq \mu}$.

Algorithm 5.4 IncrementalReductionH $(\boldsymbol{a}, \boldsymbol{f},(\mathbb{F}(t), \sigma), \delta, \boldsymbol{B})$

Input: A weak $(m-1)$-solvable $\Pi \Sigma$-ext. $(\mathbb{F}(t), \sigma)$ of $(\mathbb{G}, \sigma)$ with $\mathbb{K}:=$ const $_{\sigma} \mathbb{G}$ and $\mathbb{F}:=\mathbb{G}\left(t_{1}\right) \ldots\left(t_{e}\right)$; a bounding matrix $\boldsymbol{B}$ of length $e+1$ for $\mathbb{F}, \delta \in \mathbb{N}_{0} \cup\{-1\}$; $\mathbf{0} \neq \boldsymbol{a} \in \mathbb{F}[t]^{m}$ with $l:=\|\boldsymbol{a}\|$, and $\boldsymbol{f} \in \mathbb{F}[t]_{l+\delta}^{n}$.

Output: A basis of a subspace of $\mathrm{V}\left(\boldsymbol{a}, \boldsymbol{f}, \mathbb{F}[t]_{d}\right)$ over $\mathbb{K}$.

Exactly the same lines as in Algorithm 4.4, but replacing lines (3), (5) and (8) with:

(3) Compute $\tilde{\mathcal{B}}:=$ SolveSolutionSpaceH $\left(\tilde{\boldsymbol{a}}_{\delta}, \tilde{\boldsymbol{f}}_{\delta},(\mathbb{F}, \sigma), \boldsymbol{B}\right)$.

(5) Compute $\mathcal{B}:=\operatorname{IncrementalReductionH}(\boldsymbol{a},(0),(\mathbb{F}(t), \sigma), \delta-1, \boldsymbol{B})$. Extract a basis, say $H=\left\{g_{1}, \ldots, g_{\mu}\right\}$, for $\{g \mid(c, g) \in \mathbb{V}\}$ where $\mathbb{V}$ is generated by $\mathcal{B}$.

(8) Compute $\mathcal{B}:=\operatorname{IncrementalReductionH}\left(\boldsymbol{a}, \boldsymbol{f}_{\delta-1},(\mathbb{F}(t), \sigma), \delta-1, \boldsymbol{B}\right)$.

Following the explanations in Subsection 3.3 it is easy to see that the above algorithms compute a set $\mathcal{B}$ which spans a subspace $\mathbb{V}$ of $\mathrm{V}\left(\boldsymbol{a}, \boldsymbol{f}, \mathbb{G}\left(t_{1}\right) \ldots\left(t_{e}\right)\right)$. Together with [39, Thm. 6.2] it follows even that the elements of $\mathcal{B}$ are linearly independent, i.e., $\mathcal{B}$ is a basis of $\mathbb{V}$.

Example 5.5 (Cont. Exp. 3.9) By choosing the bounding matrix $B=\left(\begin{array}{lll}0 & 0 & 0 \\ 0 & 0 & 0\end{array}\right)$ we compute with SolveSolutionSpaceH $(\boldsymbol{a}, \boldsymbol{f},(\mathbb{Q}(n)(k)(b)(h), \sigma), \boldsymbol{B})$ a basis $\mathcal{B}_{1}$ of a subspace of $\mathrm{V}(\boldsymbol{\alpha}, \boldsymbol{\phi}, \mathbb{Q}(n)(k)(b)(h))$. This can be seen as follows.

- Since $h$ is a $\Sigma$-extension, we apply [25, Alg. 2] and compute the denominator bound given in Example 3.5; this gives $\boldsymbol{a}$ and $\boldsymbol{f}$. The last column in $\boldsymbol{B}$ defines the approximated degree bound $0+\max (\|\boldsymbol{f}\|-\|\boldsymbol{a}\|, 1)=1$. Hence we arrive at the coefficient problem $\mathrm{V}\left(\tilde{\boldsymbol{a}}_{1}, \tilde{\boldsymbol{f}}_{1}, \mathbb{Q}(n)(k)(b)\right)$ as given in Example 3.9, which we try to solve as follows.

- We compute the weak denominator bound $d^{\prime}=1$ for $\mathrm{V}\left(\tilde{\boldsymbol{a}}_{1}, \tilde{\boldsymbol{f}}_{1}, \mathbb{Q}(n)(k)(b)\right)$ by using [25, Alg. 2]. The second column in $\boldsymbol{B}$ gives the approximated denominator bound $1 b^{0}$ and the approximated degree bound $0+\max \left(\left\|\tilde{\boldsymbol{f}}_{1}\right\|-\left\|\tilde{\boldsymbol{a}}_{1}\right\|, 0\right)=1$. Afterwards we apply the incremental reduction for $\mathrm{V}\left(\tilde{\boldsymbol{a}}_{1}, \tilde{\boldsymbol{f}}_{1}, \mathbb{Q}(n)(k)(b)\right)=$ $\mathrm{V}\left(\tilde{\boldsymbol{a}}_{1}, \tilde{\boldsymbol{f}}_{1}, \mathbb{Q}(n)(k)[b]_{1}\right)$.

- This time we have algorithms in hand that solve the corresponding coefficient problems in $(\mathbb{Q}(n)(k), \sigma)$; see Theorem 4.6 ; therefore the first column in $\boldsymbol{B}$ is not considered. 
To this end, we arrive at the linearly independent solutions $\tilde{\mathcal{B}}_{1}$ given in Example 3.9. Given $\tilde{\mathcal{B}}_{1}$ we obtain the coefficient problem $\mathrm{V}\left(\tilde{\boldsymbol{a}}_{0}, \tilde{\boldsymbol{f}}_{0}, \mathbb{Q}(n)(k)(b)\right)$ whose solutions $\tilde{\mathcal{B}}_{0}$ are obtained as outlined for $\tilde{\mathcal{B}}_{1}$. Finally, we arrive at $\mathcal{B}_{1}$ as explained in Example 3.9.

Remark 5.6 The following remarks are adequate.

- For various applications it suffices to find only one non-trivial solution of problem $P L D E$. Hence one can stop looking for an appropriate bounding matrix when such a solution is found. Typical examples are the computation of all sum solutions, see [8-10,32], or the application of (creative) telescoping for $\partial$-finite summand terms; see Example 2.4 .

- As mentioned in Remark 4.8, denominator and degree bound algorithms have been developed and implemented in Sigma for various special cases; in particular for $\Sigma^{*}$-extensions. If one runs into these cases, the given algorithms are used instead of the bounding matrix mechanism.

- In our Sigma implementation we provide for simplicity the bounding matrix $\left(\begin{array}{ccc}x_{1} & \ldots & x_{e} \\ c & \ldots & c\end{array}\right) \in \mathbb{N}_{0}^{2 \times e}$ where $x_{i}=c$ if $t_{i}$ is a $\Pi$-extension, and $x_{i}=0$ otherwise. It turned out that with the choice $c=1$ one computes already a basis of $\mathrm{V}(\boldsymbol{a}, \boldsymbol{f}, \mathbb{E})$ in most situations. Otherwise, a small variation of $c$ gave us immediately the whole solution space; in Example 5.5 we chose $c=0$.

To this end, we show that there exists a bounding matrix $\boldsymbol{B}$ such that our algorithms compute all solutions of problem PLDE.

TheOREM 5.7 Let $(\mathbb{E}, \sigma)$ with $\mathbb{E}:=\mathbb{G}\left(t_{1}\right) \ldots\left(t_{e}\right)$ be a weak $(m-1)$-solvable $\Pi \Sigma$-extension of $(\mathbb{G}, \sigma), \mathbf{0} \neq \boldsymbol{a} \in \mathbb{E}^{m}$ and $\boldsymbol{f} \in \mathbb{E}^{n}$. Then there exists a bounding matrix $\boldsymbol{B}$ of length e for $\mathbb{E}$ such that $\operatorname{SolveSolutionSpaceH}(\boldsymbol{a}, \boldsymbol{f},(\mathbb{E}, \sigma), \boldsymbol{B})$ computes a basis of $\mathrm{V}(\boldsymbol{a}, \boldsymbol{f}, \mathbb{E})$.

Proof The theorem follows by proving the following stronger result. Let

$$
S:=\left\{\left(\boldsymbol{a}_{1}, \boldsymbol{f}_{1}\right), \ldots,\left(\boldsymbol{a}_{k}, \boldsymbol{f}_{k}\right)\right\}
$$

with $\mathbf{0} \neq \boldsymbol{a}_{i} \in \mathbb{E}^{m_{i}}$ and $\boldsymbol{f}_{i} \in \mathbb{E}^{n_{i}}$ for some $m_{i}, n_{i} \geq 1$. Then there is a bounding matrix $\boldsymbol{B}$ of length $e$ for $\mathbb{E}=\mathbb{G}\left(t_{1}\right) \ldots\left(t_{e}\right)$ with the following property. For any $1 \leq i \leq k$ and any matrix $\boldsymbol{M} \in \mathbb{K}^{m_{i} \times m_{i}}$ one can compute a basis of $\mathrm{V}\left(\boldsymbol{a}_{i}, \boldsymbol{M} \boldsymbol{f}_{i}, \mathbb{F}\left(t_{e}\right)\right)$ by executing the algorithm SolveSolutionSpaceH $\left(\boldsymbol{a}_{i}, \boldsymbol{M} \boldsymbol{f}_{i},\left(\mathbb{F}\left(t_{e}\right), \sigma\right), \boldsymbol{B}\right)$. Then the theorem follows by considering the special case $\boldsymbol{M}=\boldsymbol{I} \boldsymbol{d}_{n_{1}}$ and $k=1$.

If $e=0$, take ( $)$ as bounding matrix, and the theorem holds. Otherwise, assume $e \geq 1$, set $\mathbb{F}:=\mathbb{G}\left(t_{1}\right) \ldots\left(t_{e-1}\right)$ and assume that for the $\Pi \Sigma$-extension $(\mathbb{F}, \sigma)$ of $(\mathbb{G}, \sigma)$ the more general statement has been proven. Let $S$ be as above, i.e., $\mathbf{0} \neq \boldsymbol{a}_{i} \in \mathbb{F}\left(t_{e}\right)^{m_{i}}$ and $\boldsymbol{f}_{i} \in \mathbb{F}\left(t_{e}\right)^{n_{i}}$.

We proceed as in Algorithm 5.3. Namely, we adapt $\left(\boldsymbol{a}_{i}, \boldsymbol{f}_{i}\right)$, as it is performed 
in line (2) to $\left(\boldsymbol{a}_{i}^{\prime}, \boldsymbol{f}_{i}^{\prime}\right)$. For any $1 \leq i \leq k$ with $\boldsymbol{a}_{i}^{\prime} \in \mathbb{F}\left(t_{e}\right)^{1}$ we obtain a basis of $\mathrm{V}\left(\boldsymbol{a}_{i}^{\prime}, \boldsymbol{f}_{i}^{\prime}, \mathbb{F}\left(t_{e}\right)\right)$ in line (3). Therefore we can restrict $S$ to those $\boldsymbol{a}_{i}^{\prime}$ with $\boldsymbol{a}_{i}^{\prime} \notin \mathbb{F}\left(t_{e}\right)^{1}$ and write

$$
S:=\left\{\left(\boldsymbol{a}_{1}^{\prime}, \boldsymbol{f}_{1}^{\prime}\right), \ldots,\left(\boldsymbol{a}_{k^{\prime}}^{\prime}, \boldsymbol{f}_{k^{\prime}}^{\prime}\right)\right\}
$$

for some $k^{\prime} \leq k$. If $k^{\prime}=0$ we are done. Otherwise suppose $k^{\prime}>0$. Let $d_{i}^{\prime} \in \mathbb{F}\left[t_{e}\right]^{*}$ for $1 \leq i \leq k^{\prime}$ be the computed weak denominator bound of $\mathrm{V}\left(\boldsymbol{a}_{i}^{\prime}, \boldsymbol{f}_{i}^{\prime}, \mathbb{F}\left(t_{e}\right)\right)$. Then take $x_{i} \in \mathbb{N}_{0}$ such that $d_{i}^{\prime} t_{e}^{x_{i}}$ is a denominator bound of $\mathrm{V}\left(\boldsymbol{a}_{i}^{\prime}, \boldsymbol{f}_{i}^{\prime}, \mathbb{F}\left(t_{e}\right)\right)$. Now we set $x:=\max \left(x_{1}, \ldots, x_{k^{\prime}}\right)$. Note that if $t_{e}$ is a $\Sigma$ extension, then $x_{i}=0$ for all $1 \leq i \leq k^{\prime}$ and hence $x=0$. Furthermore $d_{i}:=d_{i}^{\prime} t_{e}^{x}$ is a denominator bound of $\mathrm{V}\left(\boldsymbol{a}_{i}^{\prime}, \boldsymbol{f}_{i}^{\prime}, \mathbb{F}\left(t_{e}\right)\right)$ for all $1 \leq i \leq k^{\prime}$. Next adapt $\left(\boldsymbol{a}_{i}^{\prime}, \boldsymbol{f}_{i}^{\prime}\right)$ for the denominator bound $d_{i}$ to $\left(\boldsymbol{a}_{i}^{\prime \prime}, \boldsymbol{f}_{i}^{\prime \prime}\right)$ as it is performed in line (5). Then take a $y$ such that $b_{i}:=y+\max \left(\left\|\boldsymbol{f}_{i}^{\prime \prime}\right\|-\left\|\boldsymbol{a}_{i}^{\prime \prime}\right\|,\left\|d_{i}\right\|\right)$ is a degree bound of $\mathrm{V}\left(\boldsymbol{a}_{i}^{\prime \prime}, \boldsymbol{f}_{i}^{\prime \prime}, \mathbb{F}\left[t_{e}\right]\right)$ for all $i$ with $1 \leq i \leq k^{\prime}$. With those degree bounds $b_{i}$ we consider the incremental reductions of $\left(\boldsymbol{a}_{i}^{\prime \prime}, \boldsymbol{f}_{i}^{\prime \prime}, \mathbb{F}\left[t_{e}\right]_{b_{i}}\right)$ for all $1 \leq i \leq k^{\prime}$ with its coefficients problems, say

$$
S_{i}:=\left\{\left(\boldsymbol{a}_{i b_{i}}^{\prime \prime}, \boldsymbol{f}_{i b_{i}}^{\prime \prime}\right), \ldots,\left(\boldsymbol{a}_{i 0}^{\prime \prime}, \boldsymbol{f}_{i 0}^{\prime \prime}\right)\right\}
$$

Then by our induction assumption there is a bounding matrix $\boldsymbol{B}_{0} \in \mathbb{N}_{0}^{2 \times(e-1)}$ of length $e-1$ for $\mathbb{F}$ such that for all $1 \leq i \leq k^{\prime}$, all $\boldsymbol{M} \in \mathbb{K}^{n_{i} \times n_{i}}$ and all $(\boldsymbol{\alpha}, \boldsymbol{\phi}) \in$ $S_{i}$ one can compute a basis of $\mathrm{V}(\boldsymbol{\alpha}, \boldsymbol{M} \boldsymbol{\phi}, \mathbb{F})$ by executing the algorithm SolveSolutionSpaceH $\left(\boldsymbol{\alpha}, \boldsymbol{M} \boldsymbol{\phi},(\mathbb{F}, \sigma), \boldsymbol{B}_{0}\right)$. Hence by Proposition 3.13 one can compute a basis of the vector space $\mathrm{V}\left(\boldsymbol{a}_{i}^{\prime \prime}, \boldsymbol{M} \boldsymbol{f}_{i}^{\prime \prime}, \mathbb{F}\left[t_{e}\right]_{b}\right)$ for all $1 \leq i \leq k^{\prime}$ and all $\boldsymbol{M} \in \mathbb{K}^{n_{i} \times n_{i}}$ by calling IncrementalReductionH $\left(\boldsymbol{a}_{i}^{\prime \prime}, \boldsymbol{M} \boldsymbol{f}_{i}^{\prime \prime},(\mathbb{F}, \sigma), b_{i}, \boldsymbol{B}_{0}\right)$. Moreover, by Lemma $3.11 b_{i}$ is a degree bound of $\mathrm{V}\left(\boldsymbol{a}_{i}^{\prime \prime}, \boldsymbol{M} \boldsymbol{f}_{i}^{\prime \prime}, \mathbb{F}\left[t_{e}\right]\right)$ and $d_{i}$ is a denominator bound of $\mathrm{V}\left(\boldsymbol{a}_{i}^{\prime}, \boldsymbol{M} \boldsymbol{f}_{i}^{\prime}, \mathbb{F}\left[t_{e}\right]\right)$ for any $\boldsymbol{M} \in \mathbb{K}^{n_{i} \times n_{i}}$. Summarizing, by using the bounding matrix $\boldsymbol{B}:=\boldsymbol{B}_{0 \wedge}\left(\begin{array}{l}x \\ y\end{array}\right)$ of length $e$ for $\mathbb{F}\left(t_{e}\right)$ we compute for any $1 \leq i \leq k$ and any matrix $\boldsymbol{M} \in \mathbb{K}^{n_{i} \times n_{i}}$ a basis of $\mathrm{V}\left(\boldsymbol{a}_{i}, \boldsymbol{M} \boldsymbol{f}_{i}, \mathbb{F}\left(t_{e}\right)\right)$ as claimed above. This concludes the induction step.

Note that the proof works for any other choice of (18) as long as $b$ is increased when $y$ is increased. Moreover we point out that our proof does not provide an algorithm to compute such a bounding matrix. Hence we have to loop over the possible values of the bounding matrix. Then after finitely many steps the set of the already derived solutions will stabilize. Summarizing, we obtain

THEOREM 5.8 Let $(\mathbb{F}, \sigma)$ be a weak $(m-1)$-solvable $\Pi \Sigma$-extension of $(\mathbb{G}, \sigma)$. Then there is a method that allows one to search for all solutions of problem PLDE in a systematic fashion. In particular, this holds if $(\mathbb{F}, \sigma)$ is a $\Pi \Sigma$ field over a $\sigma$-computable constant field. 


\section{Conclusion}

We have presented a general framework that provides tools to solve parameterized linear difference equations in $\Pi \Sigma$-extensions. Restricting to $\Pi \Sigma$-fields, we obtain an algorithm that finds all solutions of parameterized linear difference equations by iterative application; see Theorem 5.8. Moreover, if problems $\operatorname{Den} B$ and $\operatorname{Deg} B$ can be solved, we obtain an algorithm that finds all such solutions by only one execution; see Theorem 4.2. This special case is possible if we restrict ourself to first order linear difference equations; see Theorem 4.7. In order to apply this desirable algorithm for the higher order case, further investigations are necessary which extend the bounds given in $[10,25,26]$.

Note that our algorithmic machinery can be applied for more general difference fields than $\Pi \Sigma$-fields. For instance, in [29] we have obtained an algorithm that can solve parameterized first order linear difference equations in a $\Pi \Sigma$ extension over $(\mathbb{G}, \sigma)$ where $(\mathbb{G}, \sigma)$ is a free difference field. This enables us to apply telescoping and creative telescoping on summands with unspecified sequences. Our framework might be helpful to develop these algorithms further in order to handle not only the first order case, but also the higher order case.

Acknowledgement. I would like to thank the anonymous referee for his valuable comments.

\section{References}

[1] Petkovšek, M., Wilf, H. S. and Zeilberger, D., 1996, A=B. A. K. Peters, Wellesley, MA.

[2] Abramov, S. A., 1989, Rational solutions of linear differential and difference equations with polynomial coefficients. U.S.S.R. Comput. Math. Math. Phys., 29, 7-12.

[3] Abramov, S. A., 1989, Problems in computer algebra that are connected with a search for polynomial solutions of linear differential and difference equations. Moscow Univ. Comput. Math. Cybernet., 3, 63-68.

[4] Petkovšek, M., 1992, Hypergeometric solutions of linear recurrences with polynomial coefficients. J. Symbolic Comput., 14, 243-264.

[5] Hoeij, M. van., 1998, Rational solutions of linear difference equations. In O. Gloor (Ed) Proc. ISSAC'98 (ACM Press), 120-123.

[6] Abramov, S. A., Bronstein, M. and Petkovšek, M., 1995, On polynomial solutions of linear operator equations. In T. Levelt (Ed) Proc. ISSAC'95 (New York, ACM Press), 290-296.

[7] Abramov, S. A., 1995, Rational solutions of linear difference and $q$-difference equations with polynomial coefficients. In T. Levelt (Ed) Proc. ISSAC'95 (New York, ACM Press), 285-289.

[8] Abramov, S. A. and Petkovšek, M., 1994, D'Alembertian solutions of linear differential and difference equations. In J. von zur Gathen (Ed) Proc. ISSAC'94 (Baltimore, ACM Press), 169174 .

[9] Hendriks, P. A. and Singer, M. F., 1999, Solving difference equations in finite terms. J. Symbolic Comput., 27, 239-259.

[10] Schneider, C., 2001, Symbolic summation in difference fields. Technical Report 01-17, RISC-Linz, J. Kepler University, PhD Thesis.

[11] Chyzak, F., 2000, An extension of Zeilberger's fast algorithm to general holonomic functions. Discrete Math., 217, 115-134.

[12] Gosper, R. W., 1978, Decision procedures for indefinite hypergeometric summation. Proc. Nat. Acad. Sci. U.S.A., 75, 40-42.

[13] Zeilberger, D., 1990, A fast algorithm for proving terminating hypergeometric identities. Discrete Math., 80, 207-211. 
[14] Petkovšek, M., 1994, A generalization of Gosper's algorithm. Discrete Math., 134, 125-131.

[15] Paule, P. and Schorn, M., 1995, A Mathematica version of Zeilberger's algorithm for proving binomial coefficient identities. J. Symbolic Comput., 20, 673-698, 1995.

[16] Paule, P. and Riese, A., 1997, A Mathematica q-analogue of Zeilberger's algorithm based on an algebraically motivated aproach to $q$-hypergeometric telescoping. In M. Ismail and M. Rahman (Eds) Special Functions, q-Series and Related Topics, volume 14 (Fields Institute Toronto, AMS), 179-210.

[17] Bauer, A. and Petkovšek, M., 1999, Multibasic and mixed hypergeometric Gosper-type algorithms. Journal of Symbolic Computation, 28, 711-736.

[18] Karr, M., 1981, Summation in finite terms. J. ACM, 28, 305-350.

[19] Karr, M., 1985, Theory of summation in finite terms. J. Symbolic Comput., 1, 303-315.

[20] Schneider, C., 2005, Product representations in $\Pi \Sigma$-fields. Annals of Combinatorics, 9, $75-99$.

[21] Schneider, C., 2000, An implementation of Karr's summation algorithm in Mathematica. Sém. Lothar. Combin., S43b, 1-10.

[22] Risch, R., 1970, The solution to the problem of integration in finite terms. Bull. Amer. Math. Soc., 76, 605-608.

[23] Bronstein, M., 2000, On solutions of linear ordinary difference equations in their coefficient field. J. Symbolic Comput., 29, 841-877.

[24] Singer, M. F., 1991, Liouvillian solutions of linear differential equations with Liouvillian coefficients. J. Symbolic Comput., 11, 251-274.

[25] Schneider, C., 2004, A collection of denominator bounds to solve parameterized linear difference equations in $\Pi \Sigma$-extensions. In D. Petcu, et al. (Eds) Proc. SYNASC04, 6th Internat. (Timisoara, Mirton Publishing), 269-282.

[26] Schneider, C., 2005, Degree bounds to find polynomial solutions of parameterized linear difference equations in $\Pi \Sigma$-fields. To appear in Appl. Algebra Engrg. Comm. Comput.

[27] Schneider, C., 2004, Symbolic summation with single-nested sum extensions. In J. Gutierrez (Ed) Proc. ISSAC'04 (Santander, ACM Press), 282-289.

[28] Schneider, C., 2005, Finding Telescopers with Minimal Depth for Indefinite Nested Sum and Product Expressions. To appear in Proc. ISSAC'05.

[29] Kauers, M. and Schneider, C., 2004, Indefinite summation with unspecified sequences. SFBReport 2004-13, J. Kepler University, Linz.

[30] Wegschaider, K., 1997, Computer generated proofs of binomial multi-sum identities. Diploma thesis, RISC Linz, Johannes Kepler University.

[31] Riese, A., 2003, qMultisum - A package for proving $q$-hypergeometric multiple summation identities. J. Symbolic Comput., 35, 349-377.

[32] Schneider, C., 2004, The summation package Sigma: Underlying principles and a rhombus tiling application. Discrete Math. Theor. Comput. Sci., 6, 365-386.

[33] Schneider, C., 2005, A new Sigma approach to multi-summation. Advances in Applied Math., Special Issue Dedicated to Dr. David P. Robbins, 34, 740-767.

[34] Driver, K., Prodinger, H., Schneider, C. and Weideman, A., 2005, Padé approximations to the logarithm II: Identities, recurrences, and symbolic computation. To appear in Ramanujan J.

[35] Driver, K., Prodinger, H., Schneider, C. and Weideman, A, 2005, Padé approximations to the logarithm III: Alternative methods and additional results. To appear in Ramanujan J.

[36] Andrews, G. E., Paule, P. and Schneider, C., 2005, Plane partitions VI: Stembridge's TSPP Theorem. Advances in Applied Math., Special Issue Dedicated to Dr. David P. Robbins, 34, 709-739.

[37] Paule, P. and Schneider, C., 2003, Computer proofs of a new family of harmonic number identities. Adv. in Appl. Math., 31, 359-378.

[38] Put, M. van der and Singer, M. F., 1997, Galois Theory of Difference Equations. V. 1666 in Lecture Notes in Mathematics. Springer-Verlag.

[39] Schneider, C., 2002, Solving parameterized linear difference equations in $\Pi \Sigma$-fields. SFB-Report 02-19, J. Kepler University, Linz.

[40] Cohn, R. M., 1965, Difference Algebra. Interscience Publishers, John Wiley \& Sons.

[41] Krattenthaler, C. and Rivoal, T., 2004, Hypergéométrie et fonction zêta de Riemann. Preprint.

[42] Hoeij, M. van, 1999, Finite singularities and hypergeometric solutions of linear recurrence equations. J. Pure Appl. Algebra, 139, 109-131. 